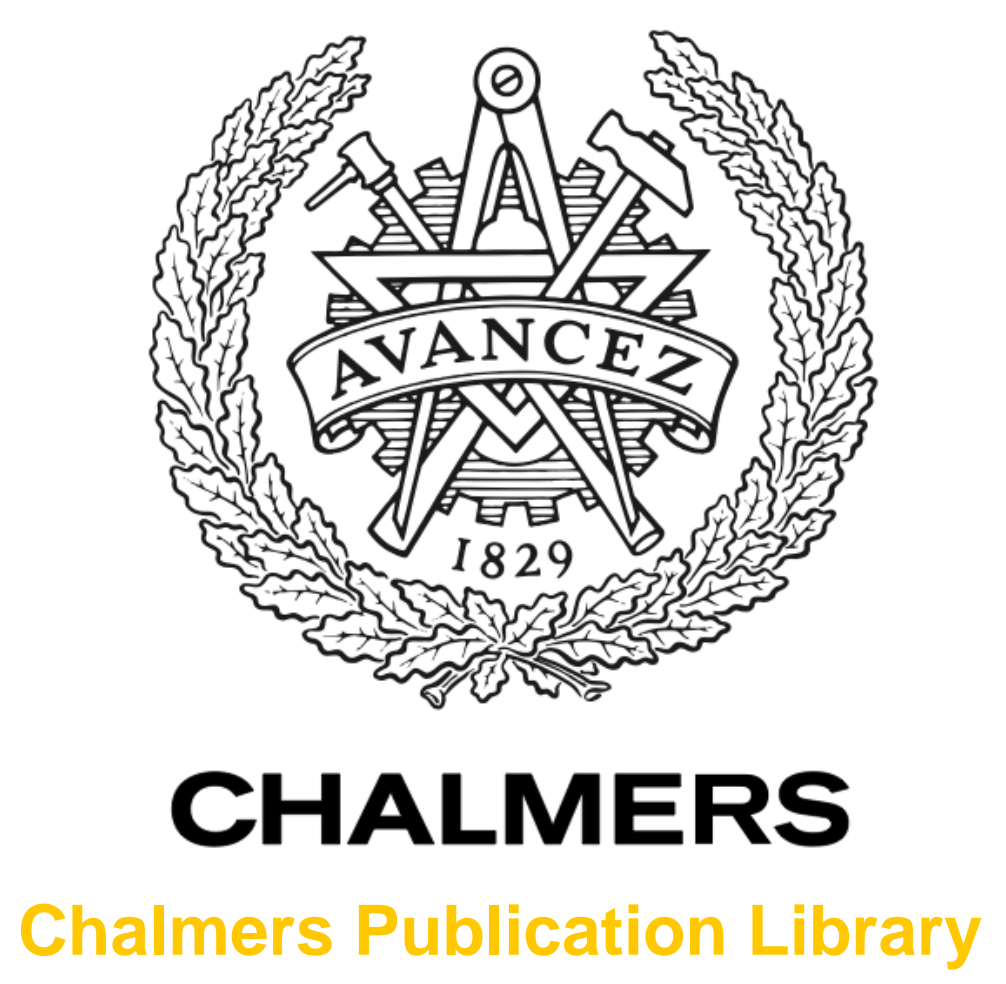

A Low Reynolds Number Partially-Averaged Navier-Stokes Model for Turbulence

This document has been downloaded from Chalmers Publication Library (CPL). It is the author's version of a work that was accepted for publication in:

International Journal of Heat and Fluid Flow (ISSN: 0142-727X)

Citation for the published paper:

Ma, J. ; Peng, S. ; Davidson, L. (2011) "A Low Reynolds Number Partially-Averaged

Navier-Stokes Model for Turbulence". International Journal of Heat and Fluid Flow, vol.

32(3), pp. 652-669.

http://dx.doi.org/10.1016/j.ijheatfluidflow.2011.02.

Downloaded from: http://publications.lib.chalmers.se/publication/129779

Notice: Changes introduced as a result of publishing processes such as copy-editing and formatting may not be reflected in this document. For a definitive version of this work, please refer to the published source. Please note that access to the published version might require a subscription.

Chalmers Publication Library (CPL) offers the possibility of retrieving research publications produced at Chalmers University of Technology. It covers all types of publications: articles, dissertations, licentiate theses, masters theses, conference papers, reports etc. Since 2006 it is the official tool for Chalmers official publication statistics. To ensure that Chalmers research results are disseminated as widely as possible, an Open Access Policy has been adopted.

The CPL service is administrated and maintained by Chalmers Library. 


\title{
A Low Reynolds Number Variant of Partially-Averaged Navier-Stokes Model for Turbulence
}

\author{
J.M. Ma ${ }^{1,2}$, S.-H. Peng ${ }^{2,3}$, L. Davidson ${ }^{2}$, and F.J. Wang ${ }^{1}$ \\ ${ }^{1}$ College of Water Conservancy \& Civil Engineering, China Agricultural University, \\ Qinghua East Road 17, Haidian District, Beijing, China \\ ${ }^{2}$ Department of Applied Mechanics, Chalmers University of Technology,SE-412 96 \\ Gothenburg, Sweden \\ ${ }^{3}$ Department of Aeronautics and Systems Technology, Swedish Defence Research Agency \\ (FOI), SE-16940 Stockholm, Sweden
}

\begin{abstract}
A low Reynolds number (LRN) formulation based on the Partially Averaged Navier-Stokes (PANS) modelling method is presented, which incorporates improved asymptotic representation in near-wall turbulence modelling. The effect of near-wall viscous damping can thus be better accounted for in simulations of wall-bounded turbulent flows. The proposed LRN PANS model uses an LRN $k-\varepsilon$ model as the base model and introduces directly its model functions into the PANS formulation. As a result, the inappropriate wall-limiting behavior inherent in the original PANS model is corrected. An interesting feature of the PANS model is that the turbulent Prandtl numbers in the $k$ and $\varepsilon$ equations are modified compared to the base model. It is found that
\end{abstract}

\footnotetext{
Email address: jiameima@gmail.com ${ }^{1,2}$, peng@foi.se $\mathrm{s}^{2,3}$, lada@chalmers.se ${ }^{2}$, wangf j@cau.edu. $\mathrm{cn}^{1}$ (J.M. Ma ${ }^{1,2}$, S.-H. Peng ${ }^{2,3}$, L. Davidson ${ }^{2}$, and F.J. Wang ${ }^{1}$ )
} 
this modification has a significant effect on the modelled turbulence. The proposed LRN PANS model is scrutinized in computations of decaying grid turbulence, turbulent channel flow and periodic hill flow, of which the latter has been computed at two different Reynolds numbers of $R e=10600$ and 37000 . In comparison with available DNS, LES or experimental data, the LRN PANS model produces improved predictions over the standard PANS model, particularly in the near-wall region and for resolved turbulence statistics. Furthermore, the LRN PANS model gives similar or better results - at a reduced CPU time - as compared to the Dynamic Smagorinsky model.

\section{Introduction}

The PANS model was developed with an intention to smoothly simulate turbulent flows using a hierarchic rank of modelling approaches from RANS to Direct Numerical Simulation (DNS). The model was derived from a parent RANS $k-\varepsilon$ model based on the ratio between modelled and total turbulence quantities. Its formulation is closely related to two parameters: the unresolved-to-total ratio of turbulence kinetic energy $\left(f_{k}\right)$ and of its dissipation $\left(f_{\varepsilon}\right)$ (Girimaji, 2005). The resulting PANS model equations hold the same form as in the parent RANS $k-\varepsilon$ model but the model coefficients are different. It is thus easy to implement the model in an existing RANS solver, where only the model coefficients need to be changed in relation to 
the choices of $f_{k}$ and $f_{\varepsilon}$.

With $f_{k}=1.0$, for example, the PANS model will render a RANS solution. Provided that a sufficiently fine grid is used to resolve all turbulent scales, on the other hand, setting $f_{k}=0$ will remove all modelling and give a DNS solution. Setting a value between 0 and 1 for $f_{k}$, the PANS model may produce partially-resolved turbulent structures and leaving the un-resolved part modelled. With a successively refined grid and a correspondingly reduced value of $f_{k}$, the resolved turbulence energy increases with decreasing modelling effects. On a LES-comparable grid, the PANS model behaves in a manner similar to an SGS model, yet being distinct from typical LES in, most noticeably, that the flow decomposition is based on turbulence energy content rather than explicit wave-number cutoff (Girimaji, 2006).

The PANS model has been evaluated in typical turbulent separating flows. Girimaji (2006) applied the PANS model to flows past a square cylinder and over a surface-mounted cube. The model was further investigated by Girimaji and Lavin (2006) in simulations of a turbulent square jet, demonstrating reasonable capabilities in capturing jet physics. Basu et al. (2007) employed the PANS model to a cavity flow with varying values of $f_{k}$ as a function of local grid spacing and turbulent length scale. Frendi et al. (2007) compared three modelling approaches (DES, URANS and PANS) and showed that the PANS model gives promising predictions for a turbulent flow over a backward 
facing step. In simulations of the flow past a square cylinder, Song and Park (2009) investigated the determination of parameter $f_{k}$ and proposed an equation to evaluate this parameter. It was demonstrated that, for separated turbulent flows, the PANS approach is able to yield accurate predictions on a relatively coarse grid. The influence of the PANS parameter $f_{\varepsilon}$ was also investigated, and it was shown that the range of resolved scales decreases with decreasing $f_{\varepsilon}$ (Frendi et al., 2007). Basara et al. (2009) combined the $\zeta-f$ model with the PANS model and applied it to the flow around the Ahmed body. A model similar to the PANS model is the PITM model (Schiestel and Dejoan, 2005).

The derivation of the original PANS model is stemmed from the standard RANS $k-\varepsilon$ model (Girimaji, 2005, 2006), which has its roots in the context of modelling high Reynolds number turbulence. As noted by its developer (Girimaji, 2006), the PANS model will inevitably inherit much of physics from its parent RANS closure. It is well known that the standard $k-\varepsilon$ model in the context of RANS modelling cannot be integrated directly to the wall surface because of its incorrect near-wall asymptotic behavior. This drawback is inherited by the resulting PANS model equations. In simulations of flows over a bluff body, the effect of near-wall asymptotic properties of the turbulence model may be insignificant. For attached boundary layer flows, however, appropriate modelling of near-wall turbulence may become essential to render 
an accurate representation of overall flow physics. One of the major purposes of the present paper is to develop a Low-Reynolds Number (LRN) variant of the PANS model so that it is feasible to apply the LRN PANS model to both attached and separated turbulent flows with improved near-wall behavior in the modelling. Another purpose is to investigate the effect of the turbulent Prandtl numbers in the $k$ and $\varepsilon$ equations, $\sigma_{k}$ and $\sigma_{\varepsilon}$. A unique feature of the PANS model is the modification of $\sigma_{k}$ and $\sigma_{\varepsilon}$ compared to the RANS $k$ and $\varepsilon$ equations. They are both multiplied by $f_{k}^{2} / f_{\varepsilon}$. This modification is found to have a strong effect on the modeled turbulence.

An LRN formulation of a turbulence model accounts for both viscous and wall-damping effects in the wall layer. It should enable proper modelling performance when the model is integrated to the wall surface. LRN RANS models usually employ empirical damping functions in the model equations, which ensure that viscous stresses take over turbulent Reynolds stresses at low Reynolds numbers and in the viscous sublayer adjacent to solid walls. There are a number of LRN RANS models, of which many are based on the $k-\varepsilon$ models, see e.g. Jones and Launder (1972); Hoffmann (1975); Chien (1982); Nagano and Tagawa (1990). Abe et al. (1994) (hereafter called the AKN model) further improved the LRN $k-\varepsilon$ model by Nagano and Tagawa (1990), using the Kolmogorov velocity scale, $u_{\varepsilon} \equiv(\nu \varepsilon)^{1 / 4}$, in the damping function. The AKN model was validated in simulations of turbulent flows in 
a channel and over a backward step. Hsieh and Chang (1996) applied the AKN model to pipe expansion flow with satisfactory results. Of the existing LRN $k-\varepsilon$ models, the AKN model has shown reasonable performance in modelling different flows.

The present work proposes an LRN variant of the PANS model using the LRN AKN $k-\varepsilon$ model as the base model. The derivation of the LRN PANS formulation is similar to the original PANS model (or the "standard PANS model"), but the model coefficients are adapted to the LRN effect in order to account for near-wall turbulence. The behavior of the proposed model is examined in simulations of decaying grid turbulence, fully developed turbulent flows in a clean channel and in a channel with hills mounted periodically on the bottom wall. The hill flow has been computed at two different Reynolds numbers, $R e=10600$ and 37000 , respectively. The LRN PANS model has been compared with the standard PANS model in these computations, as well as with LES using the Dynamic Smagorinsky model. The proposed LRN model has shown overall improved wall-limiting behaviors in the wall layer in comparison with the standard PANS model.

In what follows, we first present the modelling formulation in Section 2. In Section 3 the numerical methods used in the computations are briefly described, including an introduction to the test cases and computational setup. The results are then presented and discussed in Section 4, and conclusions 
are drawn in Section 5.

\section{Modelling formulation}

The PANS method uses the so-called "partial averaging" concept, which corresponds to a filtering operation for a portion of the fluctuating scales (Girimaji, 2006). The "partial-averaging" operation is assumed to be constant preserving and to commute with spatial and temporal differentiation.

For an instantaneous flow variable, $F$, we use $\bar{f}$ to denote the partiallyaveraged part, namely $\bar{f}=\mathcal{P}(F)$, where $\mathcal{P}$ denotes the partial-averaging operator. We consider incompressible flows. Applying the partial averaging to the governing equations gives

$$
\begin{gathered}
\frac{\partial \bar{u}_{i}}{\partial x_{i}}=0 \\
\frac{\partial \bar{u}_{i}}{\partial t}+\frac{\partial\left(\bar{u}_{i} \bar{u}_{j}\right)}{\partial x_{j}}=-\frac{1}{\rho} \frac{\partial \bar{p}}{\partial x_{i}}+\frac{\partial}{\partial x_{j}}\left(\nu \frac{\partial \bar{u}_{i}}{\partial x_{j}}-\tau_{i j}\right)
\end{gathered}
$$

where $\tau_{i j}$ is the central second moment resulting from the partial averaging for the nonlinear terms, that is $\tau_{i j}=\left(\mathcal{P}\left(U_{i} U_{j}\right)-\bar{u}_{i} \bar{u}_{j}\right)$, where $U_{i}$ indicates instantaneous velocity components. This term is similar to the Reynolds stress tensor resulting from the Reynolds averaging in RANS or to the subgrid-scale (SGS) stress tensor after the spatial filtering in LES. For simplicity, we also use the terminology of Reynolds stresses for the term $\tau_{i j}$ in Eq. 2.

To close the system of the partially-averaged Navier-Stokes equations, as in RANS and LES, a model is needed for $\tau_{i j}$. Girimaji (2006) proposed using 
the conventional eddy viscosity concept so that $\tau_{i j}=-2 \nu_{u} \bar{s}_{i j}$, where $\bar{s}_{i j}$ is the strain-rate tensor of the computed flow and $\nu_{u}$ is the PANS eddy viscosity.

In order to formulate the PANS eddy viscosity, Girimaji (2006) defined another two quantities, the partially-averaged turbulent kinetic energy, $k_{u}$ and its dissipation rate $\varepsilon_{u}$, so that $\nu_{u}=C_{\mu} k_{u}^{2} / \varepsilon_{u}$. In the derivation of the transport equations for $k_{u}$ and $\varepsilon_{u}$, two parameters, $f_{k}$ and $f_{\varepsilon}$, have been introduced, relating the unresolved to the resolved fluctuating scales. Parameter $f_{k}$ defines the ratio of unresolved (partially-averaged) turbulent kinetic energy $\left(k_{u}\right)$ to the total kinetic energy $(k)$, and $f_{\varepsilon}$ is the ratio between the unresolved $\left(\varepsilon_{u}\right)$ and the total $(\varepsilon)$ dissipation rates. These give

$$
k=\frac{k_{u}}{f_{k}} \text { and } \varepsilon=\frac{\varepsilon_{u}}{f_{\varepsilon}}
$$

The extent of the resolved part is now determined by $f_{k}$ and $f_{\varepsilon}$. In his PANS derivation, Girimaji $(2005,2006)$ employed the standard $k-\varepsilon$ model as the base model. The resulting model is thus termed here the Standard PANS model.

Below, we re-formulate the PANS model based on an LRN $k-\varepsilon$ model in order to attain improved near-wall asymptotic behavior. Incorporating empirical damping functions, an LRN $k-\varepsilon$ RANS model can often be cast in a general form, of which the $k$-equation can be written as

$$
\frac{\partial k}{\partial t}+\frac{\partial\left(k \bar{U}_{j}\right)}{\partial x_{j}}=\frac{\partial}{\partial x_{j}}\left[\left(\nu+\frac{\nu_{t}}{\sigma_{k}}\right) \frac{\partial k}{\partial x_{j}}\right]+P_{k}-\varepsilon
$$


and the $\varepsilon$-equation is given by

$$
\frac{\partial \varepsilon}{\partial t}+\frac{\partial\left(\varepsilon \bar{U}_{j}\right)}{\partial x_{j}}=\frac{\partial}{\partial x_{j}}\left[\left(\nu+\frac{\nu_{t}}{\sigma_{\varepsilon}}\right) \frac{\partial \varepsilon}{\partial x_{j}}\right]+C_{\varepsilon 1} f_{1} P_{k} \frac{\varepsilon}{k}-C_{\varepsilon 2} f_{2} \frac{\varepsilon^{2}}{k}
$$

where $P_{k}$ is the production term of turbulent kinetic energy

$$
P_{k}=\nu_{t}\left(\frac{\partial \bar{U}_{i}}{\partial x_{j}}+\frac{\partial \bar{U}_{j}}{\partial x_{i}}\right) \frac{\partial \bar{U}_{i}}{\partial x_{j}}
$$

In a low Reynolds number RANS $k-\varepsilon$ model, the RANS eddy viscosity, $\nu_{t}$, often takes the following form.

$$
\nu_{t}=C_{\mu} f_{\mu} \frac{k^{2}}{\varepsilon}
$$

It should be noted that the RANS mean velocity field is denoted by $\bar{U}_{i}$ in these equations. As indicated by Girimaji (2005), one should have $\bar{U}_{i}=\left\langle\bar{u}_{i}\right\rangle$, with the angular brackets indicating the time-averaged flow quantities.

Using the same damping function, $f_{\mu}$, as for the LRN model, the PANS turbulent viscosity, $\nu_{u}$, in the LRN PANS model is defined in terms of $k_{u}$ and $\varepsilon_{u}$, viz.

$$
\nu_{u}=C_{\mu} f_{\mu} \frac{k_{u}^{2}}{\varepsilon_{u}}
$$

Introducing Eq. 3 into the relation for the RANS turbulent eddy viscosity, $\nu_{t}$, Eq. 7, gives the following relation.

$$
\nu_{t}=\frac{f_{\varepsilon}}{f_{k}^{2}} \nu_{u}
$$

In the derivation of the $k_{u}$ and $\varepsilon_{u}$ equations for the LRN PANS model, the same procedure has been invoked as for the standard PANS paradigm 
(Girimaji, 2005, 2006). Parameters $f_{k}$ and $f_{\varepsilon}$ have also been assumed to be constants. Without repeating all the details of the PANS formulation, as discussed in Girimaji (2006), we directly write the resulting transport equation for $k_{u}$ in the LRN PANS model, which takes the same form as in the standard PANS model. This gives

$$
\begin{aligned}
\frac{\partial k_{u}}{\partial t}+\frac{\partial\left(k_{u} \bar{u}_{j}\right)}{\partial x_{j}} & =\frac{\partial}{\partial x_{j}}\left[\left(\nu+\frac{\nu_{u}}{\sigma_{k u}}\right) \frac{\partial k_{u}}{\partial x_{j}}\right]+P_{u}-\varepsilon_{u} \\
\sigma_{k u} & =\sigma_{k} \frac{f_{k}^{2}}{f_{\varepsilon}}
\end{aligned}
$$

where the production term, $P_{u}$, is expressed in terms of the PANS eddy viscosity, $\nu_{u}$, and the strain rate of PANS-resolved flow field, viz.

$$
P_{u}=\nu_{u}\left(\frac{\partial \bar{u}_{i}}{\partial x_{j}}+\frac{\partial \bar{u}_{j}}{\partial x_{i}}\right) \frac{\partial \bar{u}_{i}}{\partial x_{j}}
$$

Note that, in deriving Eq. 10, a relation of $P_{u}-\varepsilon_{u}=f_{k}\left(P_{k}-\varepsilon\right)$ is implied (Girimaji, 2005). With $\varepsilon=\varepsilon_{u} / f_{\varepsilon}$, this relation can be re-written as

$$
P_{k}=\frac{1}{f_{k}}\left(P_{u}-\varepsilon_{u}\right)+\frac{\varepsilon_{u}}{f_{\varepsilon}}
$$

Equation 12 has been exploited to derive the $\varepsilon_{u}$-equation in the PANS model. With an LRN model as the base model, the $\varepsilon$ equation invokes model functions, $f_{1}$ and $f_{2}$, in the production and destruction terms, which are kept in the model coefficient for the resulting $\varepsilon_{u}$ equation. This led to the following 
expression.

$$
\begin{aligned}
\frac{\partial \varepsilon_{u}}{\partial t}+\frac{\partial\left(\varepsilon_{u} \bar{u}_{j}\right)}{\partial x_{j}} & =f_{\varepsilon}\left[\frac{\partial \varepsilon}{\partial t}+\frac{\partial\left(\varepsilon \bar{u}_{j}\right)}{\partial x_{j}}\right] \\
& =f_{\varepsilon}\left\{\frac{\partial}{\partial x_{j}}\left[\left(\nu+\frac{\nu_{t}}{\sigma_{\varepsilon}}\right) \frac{\partial \varepsilon}{\partial x_{j}}\right]+C_{\varepsilon 1} f_{1} P_{k} \frac{\varepsilon}{k}-C_{\varepsilon 2} f_{2} \frac{\varepsilon^{2}}{k}\right\}
\end{aligned}
$$

The diffusion term is re-written using Eq. 3

$$
\begin{aligned}
f_{\varepsilon}\left\{\frac{\partial}{\partial x_{j}}\left[\left(\nu+\frac{\nu_{t}}{\sigma_{\varepsilon}}\right) \frac{\partial \varepsilon}{\partial x_{j}}\right]\right\} & =\frac{\partial}{\partial x_{j}}\left[\left(\nu+\frac{\nu_{t}}{\sigma_{\varepsilon}}\right) \frac{\partial \varepsilon_{u}}{\partial x_{j}}\right] \\
& =\frac{\partial}{\partial x_{j}}\left[\left(\nu+\frac{\nu_{u}}{\sigma_{\varepsilon u}}\right) \frac{\partial \varepsilon_{u}}{\partial x_{j}}\right] \\
\sigma_{\varepsilon u} & =\frac{\sigma_{\varepsilon} f_{k}^{2}}{f_{\varepsilon}}
\end{aligned}
$$

In the same way, the production and destruction terms are re-formulated as (using Eqs. 3 and 12)

$$
\begin{aligned}
f_{\varepsilon}\left\{C_{\varepsilon 1} f_{1} P_{k} \frac{\varepsilon}{k}-C_{\varepsilon 2} f_{2} \frac{\varepsilon^{2}}{k}\right\} & =C_{\varepsilon 1} f_{1} \frac{\varepsilon_{u} f_{k}}{k_{u}}\left(\frac{1}{f_{k}}\left(P_{u}-\varepsilon_{u}\right)+\frac{\varepsilon_{u}}{f_{\varepsilon}}\right)-C_{\varepsilon 2} f_{2} \frac{\varepsilon_{u}^{2} f_{k}}{f_{\varepsilon} k_{u}} \\
=C_{\varepsilon 1} f_{1} \frac{\varepsilon_{u}}{k_{u}} P_{u}-C_{\varepsilon 1} f_{1} \frac{\varepsilon_{u}^{2}}{k_{u}} & +C_{\varepsilon 1} f_{1} \frac{\varepsilon_{u}^{2} f_{k}}{k_{u} f_{\varepsilon}}-C_{\varepsilon 2} f_{2} \frac{\varepsilon_{u}^{2} f_{k}}{f_{\varepsilon} k_{u}} \\
& =C_{\varepsilon 1} f_{1} \frac{\varepsilon_{u}}{k_{u}} P_{u}-C_{\varepsilon 2}^{*} \frac{\varepsilon_{u}^{2}}{k_{u}}
\end{aligned}
$$

where

$$
C_{\varepsilon 2}^{*}=C_{\varepsilon 1} f_{1}+\frac{f_{k}}{f_{\varepsilon}}\left(C_{\varepsilon 2} f_{2}-C_{\varepsilon 1} f_{1}\right)
$$

The $\varepsilon_{u}$ equation in the LRN PANS model now takes the following form

$$
\frac{\partial \varepsilon_{u}}{\partial t}+\frac{\partial\left(\varepsilon_{u} \bar{u}_{j}\right)}{\partial x_{j}}=\frac{\partial}{\partial x_{j}}\left[\left(\nu+\frac{\nu_{u}}{\sigma_{\varepsilon u}}\right) \frac{\partial \varepsilon_{u}}{\partial x_{j}}\right]+C_{\varepsilon 1} f_{1} P_{u} \frac{\varepsilon_{u}}{k_{u}}-C_{\varepsilon 2}^{*} \frac{\varepsilon_{u}^{2}}{k_{u}}
$$

where $\sigma_{\varepsilon u}$ is given in Eq. 14 
It should be noted that, in deriving Eq. 10 and Eq. 17, the additional term related to $\left(\bar{u}_{j}-\bar{U}_{j}\right)$ has been neglected, as was done by Girimaji (2006).

The PANS equation for $k_{u}$, Eq. 10, was derived by multiplying the RANS equation for $k$ by $f_{k}$ which was assumed to be constant in space and in time. By referring to Eqs. 9 and 10, the turbulent diffusion term was obtained as

$$
\begin{aligned}
f_{k} \frac{\partial}{\partial x_{j}}\left(\frac{\nu_{t}}{\sigma_{k}} \frac{\partial k}{\partial x_{j}}\right) & =\frac{\partial}{\partial x_{j}}\left(\frac{\nu_{t}}{\sigma_{k}} \frac{\partial k_{u}}{\partial x_{j}}\right) \\
& =\frac{\partial}{\partial x_{j}}\left(\frac{\nu_{u}}{\sigma_{k u}} \frac{\partial k_{u}}{\partial x_{j}}\right)
\end{aligned}
$$

The expression on the right-hand side of Eq. 18(a) suggests that the turbulent transport for the PANS-modelled turbulent kinetic energy, $k_{u}$, is actually formulated in terms of the RANS turbulent viscosity from the base model. This is different from the turbulent diffusion in subgrid scale (SGS) modelling of LES with a one-equation $k_{s g s}$ model, which reads

$$
\frac{\partial}{\partial x_{j}}\left(\frac{\nu_{s g s}}{\sigma_{k}} \frac{\partial k_{s g s}}{\partial x_{j}}\right)
$$

In Eq. 19 the $S G S$ turbulent viscosity is invoked for the transport of $k_{s g s}$, whereas on the right-hand side of Eq. 18(a) the total (i.e. the RANS) turbulent viscosity has been used for $k_{u}$. Equation 18(a) shows that, when used as an SGS model, the modelled turbulent diffusion in the PANS formulation is a factor of $\left(\sigma_{k u} / \sigma_{k}\right)^{-1}=f_{\varepsilon} / f_{k}^{2}$ larger than in one-equation SGS models, comparing Eqs. 19, to 10 and 18(b). With $f_{\varepsilon}=1$ and $f_{k}=0.4$, for example, this factor is larger than six. The modification of the diffusion 
coefficients, $\sigma_{k u}$ and $\sigma_{\varepsilon u}$, is a unique property of the PANS model. In the PITM model (Schiestel and Dejoan, 2005), for example, the sink term in the $\varepsilon$ equation is modified, but not the diffusion term. The effect of the diffusion coefficients, $\sigma_{k u}$ and $\sigma_{\varepsilon u}$, in the $k_{u}$ and $\varepsilon_{u}$ equations will be investigated in the present work.

Equations 8, 10 and 17 form the proposed PANS formulation based on an LRN RANS $k-\varepsilon$ model. Obviously, to bring the formulation into an LRN PANS model, many existing LRN $k-\varepsilon$ models in the context of RANS and being cast in line with the general form as given in Eqs. 4-7, can be considered. In the present work, we have adopted the Abe-Kondoh-Nagano LRN $k-\varepsilon$ model (Abe et al., 1994) (the LRN AKN model) as the base model. The LRN PANS model constants thus take the following values

$$
C_{\varepsilon 1}=1.5, C_{\varepsilon 2}=1.9, \sigma_{k}=1.4, \sigma_{\varepsilon}=1.4, C_{\mu}=0.09
$$

With $f_{1}=1$, the other two model functions, $f_{\mu}$ and $f_{2}$, hold the same forms in the LRN PANS model as for the AKN LRN model. They read, respectively,

$$
\begin{gathered}
f_{\mu}=\left[1-\exp \left(-\frac{y^{*}}{14}\right)\right]^{2}\left\{1+\frac{5}{R_{t}^{3 / 4}} \exp \left[-\left(\frac{R_{t}}{200}\right)^{2}\right]\right\} \\
f_{2}=\left[1-\exp \left(-\frac{y^{*}}{3.1}\right)\right]^{2}\left\{1-0.3 \exp \left[-\left(\frac{R_{t}}{6.5}\right)^{2}\right]\right\}
\end{gathered}
$$

The variables, $R_{t}$ and $y^{*}$, are now defined in terms of $k_{u}$ and $\varepsilon_{u}$ for the LRN 
PANS model, namely,

$$
R_{t}=\frac{k_{u}^{2}}{\nu \varepsilon_{u}} \text { and } y^{*}=\frac{U_{\varepsilon} y}{\nu} \text { with } U_{\varepsilon}=\left(\varepsilon_{u} \nu\right)^{1 / 4}
$$

The model coefficients in the proposed model are different from these in the standard PANS model (Girimaji, 2006). The introduction of the damping functions has indeed enabled the resulting LRN PANS model to have improved asymptotic properties when integrated to a wall surface. Ap-

proaching the wall surface, the LRN PANS model suggests that $f_{\mu} \propto y^{-1}$. Consequently, the modelled turbulent quantities hold $\overline{u v} \propto y^{3}, k_{u} \propto y^{2}$, $\varepsilon_{u} \propto y^{0}$ and $\nu_{u} \propto y^{3}$ in the vicinity of the wall surface, as desired. It is thus expected that the resulting LRN PANS model will improve the near-wall representation of modelled turbulence and, as a result, the near-wall turbulence should also be better resolved, as compared with the standard PANS model.

\section{Computational set-up}

An incompressible, finite volume code was used (Davidson and Peng, 2003). The second-order central differencing scheme was used for spatial discretization of all terms except the convection terms in the $k_{u}$ and $\varepsilon_{u}$ equations, for which a hybrid central/upwind scheme was employed. The temporal advancement was approximated using the second-order Crank-Nicolson scheme. The numerical procedure was based on an implicit, fractional step 
technique with a multigrid pressure Poisson solver (Emvin, 1997) and a nonstaggered grid arrangement.

The proposed model was examined in computations of three different flow problems. For reference, the standard PANS model and the Dynamic Smagorinsky model were also employed in the computations.

In order to highlight any possible effect on the resolved spectral behavior due to the introduced low-Reynolds number modifications, decaying homogeneous, isotropic turbulence (DHIT) is first computed. DHIT is a typical test case for SGS turbulence models. The computational domain is a cubic box with dimensions of $2 \pi \times 2 \pi \times 2 \pi$. Two meshes are used, one with $32 \times 32 \times 32$ cells and another with $64 \times 64 \times 64$ cells. Periodic boundary conditions are prescribed at all boundaries. The time step is $5 \cdot 10^{-3}$.

The second test case is a fully-developed turbulent channel flow. Note that previous PANS simulations have usually been conducted for bluff-body flows. The test case is selected to highlight the feasibility of the PANS model in computations of attached boundary layer flows, particularly, the modelling behavior in representing near-wall turbulence. The Reynolds number is $R e_{\tau}=950$, based on the friction velocity, $u_{\tau}$, and half of the channel height, $\delta=y_{\max } / 2$. The computational domain has the dimensions of $x_{\max }=3.2$, $y_{\max }=2.0$ and $z_{\max }=1.6$. A $64 \times 80 \times 64$ mesh has been used in the streamwise $(x)$, wall-normal $(y)$ and spanwise $(z)$ directions, respectively. Table 
1 summarizes the computational parameters for the channel flow computations. DNS data was taken from the work by Hoyas and Jimenez (2008).

\begin{tabular}{c|cccc}
\hline$R e_{\tau}$ & $\Delta y^{+}$ & $\Delta x^{+}$ & $\Delta z^{+}$ & $\Delta t$ \\
\hline 950 & $0.62-103$ & 47.50 & 23.75 & $6.25 \times 10^{-4}$ \\
\hline
\end{tabular}

Table 1: Spatial resolution and time step in computations of turbulent channel flow

The third and fourth test cases concern flow separation in a channel with periodic hills mounted on the bottom wall in the streamwise direction. The periodic hill flow is characterized by turbulent flow separation, recirculation, reattachment and flow acceleration. The geometry and mesh of the hill flow are shown in Fig. 1. The computational domain starts from one hill crest and extends to the next, separated by a distance of $L=9 h$. The upper and lower sides are bounded by flat plane and curved wall surfaces, respectively. The extension in the spanwise direction is $z_{\max }=4.5 h$. This flow has been computed for two different Reynolds numbers, Re $=10600$ and 37000 . The Reynolds number is based on the hill height, $h$, and the bulk velocity, $U_{b}$, above the hill crest. For $R e=37000$, the computational mesh consists of $N_{x} \times N_{y} \times N_{z}=160 \times 160 \times 60$ cells in the streamwise, wallnormal and spanwise directions, respectively. For $R e=10600$ every second grid line is omitted in the wall-normal direction and the number of cells in 


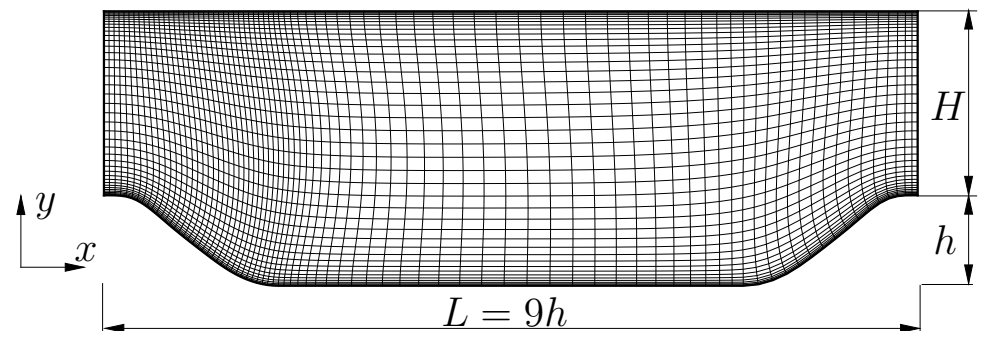

Figure 1: Schematic of the hill flow configuration and computational grid in a $2 D$ slice. The grid was plotted with every other line. $h=1.0, H=2.035 h, L=9 h, z_{\max }=4.5 h$.

the spanwise direction is reduced by almost $50 \%$, giving a computational mesh with $N_{x} \times N_{y} \times N_{z}=160 \times 80 \times 32$ cells. The time step was set to $\Delta t=6.0 \times 10^{-3}$. After 20 flow-through times, statistical analysis was made over a time period of a further 20 flow-through times. The results were also averaged in space over the spanwise direction.

For all computations of wall-bounded flows, no-slip conditions were specified on the walls for the velocity components. The values of $k_{u}$ and $\varepsilon_{u}$ on the wall surface were set by $k_{u, w}=0$ and $\varepsilon_{u, w}=2 \nu k_{u, 1} / y_{1}^{2}$, respectively, where $k_{u, 1}$ is the value of $k_{u}$ at the first near-wall node with a wall distance of $y_{1}$. Periodic boundary conditions were imposed on the streamwise and spanwise boundaries. 


\section{Results and discussion}

This section presents the computations with the LRN PANS model for DHIT, channel flow and periodic hill flow in comparison with the standard PANS model and the Dynamic Smagorinsky model of Germano et al. (1991). The main purpose is to verify the performance of the proposed LRN PANS model in turbulence-resolving simulations for both attached and separated turbulent flows. The results are compared to available DNS, LES or experimental data.

By the definition of $f_{k}$ and $f_{\varepsilon}$, it is obvious that these two parameters should vary with the grid resolution in correspondence to the resolved kinetic energy and dissipation rate. As proposed by Girimaji (2005), nonetheless, we have set $f_{\varepsilon} \equiv 1$ in all the computations. For the same grid resolution, different values of $f_{k}$ are then tested to observe the effect of this parameter on the modelling.

\subsection{Decaying homogeneous, isotropic turbulence}

The simulations for this test case are compared with the experiment by Comte-Bellot and Corrsin (1971). In the experiment the turbulence spectra were measured at three downstream locations corresponding to the times $t U_{0} / M=42,98$ and 171 . The initial velocity is obtained by inverse Fourier transformation using the experimental spectrum at $t U_{0} / M=42$. At time 
$t U_{0} / M=171$ the spectra resulting from the simulations are compared the experimental spectrum. The initial velocity field is generated by a widely used computer program from the group of Prof. Strelets in St. Petersburg.

Initial boundary conditions must also be given for $k_{u}$ and $\varepsilon_{u}$ in PANS computations. In Menter and Egorov (2010) they computed the initial $k_{u}$ and $\varepsilon_{u}$ fields by solving the steady $k_{u}$ and $\varepsilon_{u}$ transport equations using the frozen initial velocity field. It was noted however that $k_{u}$ and $\varepsilon_{u}$ (and $\nu_{u}$ ) go to infinity (Menter and Egorov, 2010). The same problem is encountered with the PANS model in our computations.

In the present work another approach is chosen to prescribe initial conditions for $k_{u}$ and $\varepsilon_{u}$. Using the initial velocity field, the resolved turbulence energy, $k_{\text {res }}=0.5\left\langle u_{i}^{\prime} u_{i}^{\prime}\right\rangle(\langle$.$\rangle denotes space averaging over the entire domain),$ is computed and the initial modelled turbulence is set as $k_{u}=f_{k} k_{\text {res }}$. The initial SGS length scale, $\ell_{s g s}$, is estimated from the Smagorinsky model as $\ell_{\text {sgs }}=C_{s} \Delta$ where $C_{s}=0.1$ and $\Delta$ is the grid spacing. The initial modelled dissipation is then approximated from $\varepsilon_{u}=C_{\mu}^{3 / 4} k_{u}^{3 / 2} / \ell_{s g s}$. Since the flow does not involve any wall boundaries and the wall distance is thus set to be infinite, the first part in the the damping functions, $f_{\mu}$ in Eq. 21 and $f_{2}$ in Eq. 22, becomes one, respectively. Consequently, $f_{\mu}$ and $f_{2}$ play a role in the modelling only as function of the turbulent Reynolds number, $R_{t}$. Obviously, $f_{2}$ approaches to 1.0 at fairly small values of $R_{t}$. For example, $f_{2}$ takes its 
instantaneous smallest value of 0.995 (with $R_{t} \approx 13$ ) at $t U_{0} / M=171$ for the fine mesh with $f_{k}=0.2$. The second part of $f_{\mu}$ yields larger values than 1.0. In the parent RANS LRN model, this part in $f_{\mu}$ was designed to entail a correct near-wall asymptotic relation of $f_{\mu} \rightarrow 1 / y$ (as $y \rightarrow 0$ ) for wall-bounded flows (Abe et al., 1994). Away from the wall, $f_{\mu}$ should be limited by 1.0 in computations of any turbulent shear flows. In consistence with this, we have thus set $f_{\mu}=1.0$ in the DHIT computations.

Figure 2 presents the predicted spectra on the two meshes at the computational time $t=2$, which corresponds to $t U_{0} / M=171$ in the experiment. Predictions using four different values of $f_{k}$ are presented, namely $f_{k}=0.2$, 0.4, 0.6 and 0.8. As can be seen, the value of $f_{k}$ hardly imposes any influence for low wavenumbers $(\kappa<6)$. For higher wavenumbers, large $f_{k}$ values give more dissipation, as expected. The effect of $f_{k}$ becomes even more significant at increasing wavenumbers when the grid is refined from $32^{3}$ to $64^{3} . f_{k}=0.6$ gives good agreement with the experiment on the coarse grid, whereas a reduced value of $f_{k}$ renders too little dissipation. On the fine mesh, both $f_{k}=0.2$ and 0.4 give reasonable agreement with the experiment. The observation is consistent with the fundamental argumentation in PANS modelling, namely, the ratio of modelled-to-total turbulence energy, $f_{k}$, is related to the grid resolution. For comparison, the result computed by the Dynamic Smagorinsky model is also included in Figure 2, which gives good 


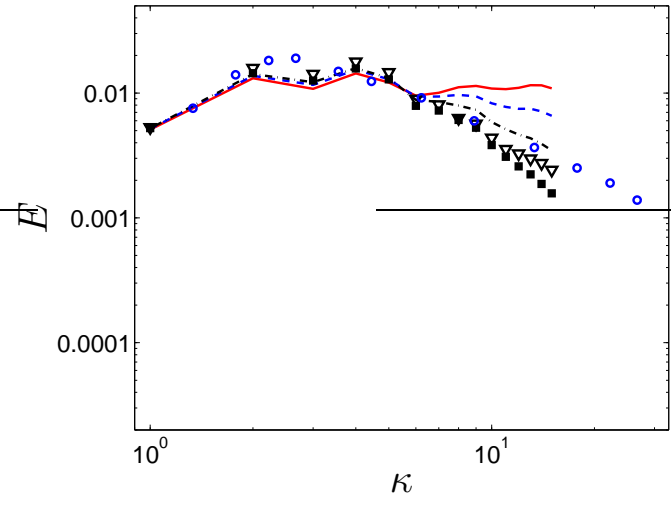

(a) $32 \times 32 \times 32$ mesh

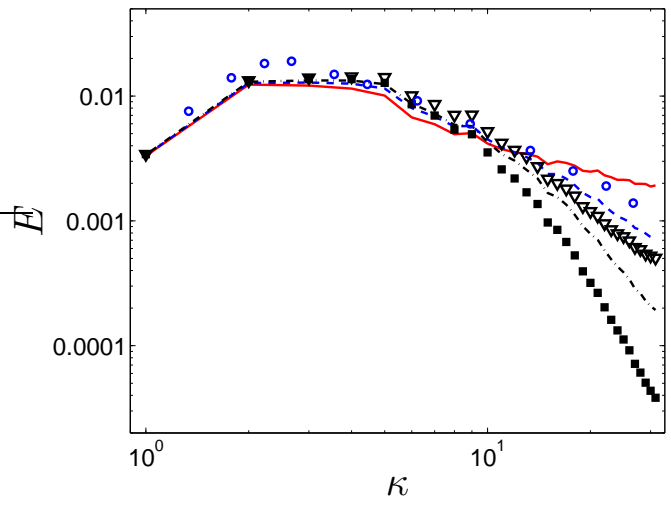

(b) $64 \times 64 \times 64$ mesh

Figure 2: DHIT using the LRN PANS model and the Dynamic Smagorinsky model. Resolved turbulence energy spectra. $\_$— $f_{k}=0.2 ; \ldots-f_{k}=0.4 ; \ldots . f_{k}=0.6$;

$f_{k}=0.8 ; \mathbf{v}$ : Dynamic Smagorinsky model; ○: Experiments by Comte-Bellot and Corrsin (1971).

agreement on the coarse mesh but is slightly more dissipative on the fine mesh at large wavenumbers.

Figures 3 and 4 present the resolved $\left(k_{\text {res }}\right)$ and the modelled $\left(k_{u}\right)$ kinetic energy versus time. With increasing $f_{k}$, as expected, $k_{u}$ increases and $k_{\text {res }}$ decreases. As shown in the predicted spectra, the dissipation is not sufficient for $f_{k}=0.2$. This has also been reflected by a relatively slow decay of the resolved turbulent kinetic energy (Fig. 3).

\subsection{Turbulent channel flow $\left(R e_{\tau}=950\right)$}

The following values were tested for $f_{k}$ in the simulation of channel flow, namely, $f_{k}=0.1,0.3,0.4,0.5,0.6$ and 1.0. As expected, the smaller the 


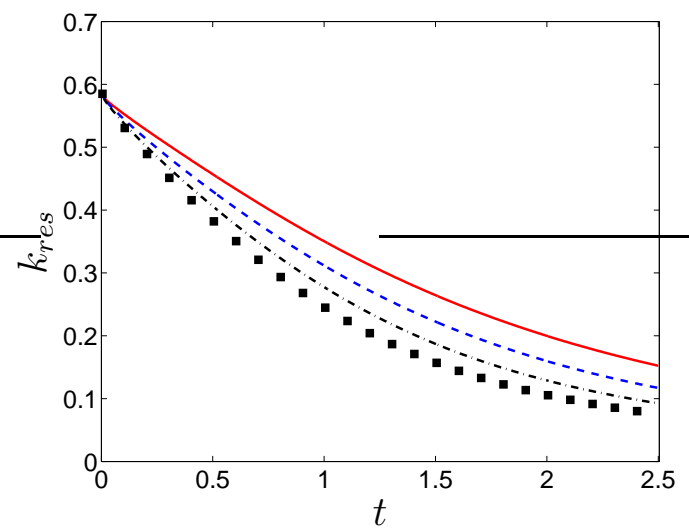

(a) $32 \times 32 \times 32$ mesh

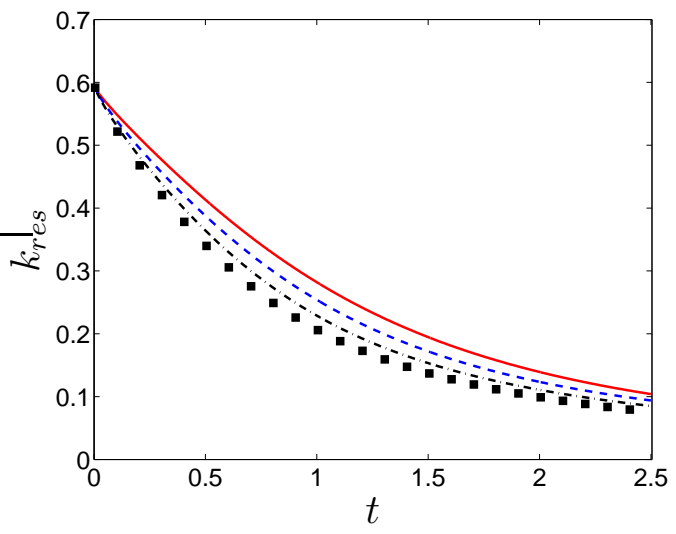

(b) $64 \times 64 \times 64$ mesh

Figure 3: DHIT using the LRN PANS model and the Dynamic Smagorinsky model. Resolved turbulence energy decaying with time., $k_{\text {res }}=0.5\left\langle u_{i}^{\prime} u_{i}^{\prime}\right\rangle$. $f_{k}=0.4 ;-\cdots: f_{k}=0.6 ; \mathbf{\square}: f_{k}=0.8$.

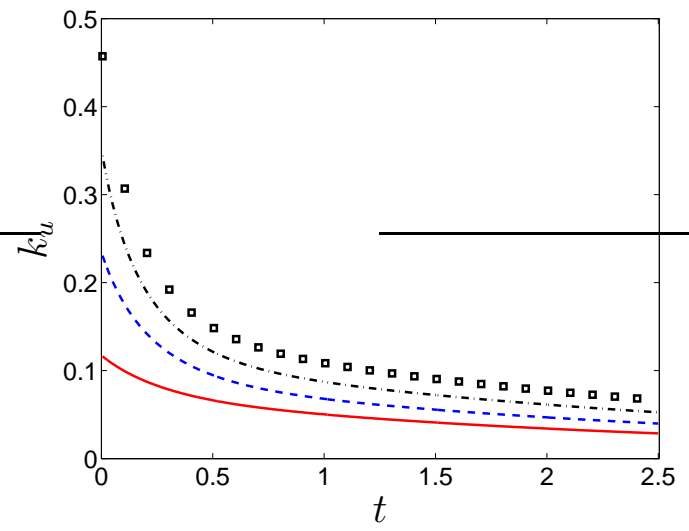

(a) $32 \times 32 \times 32$ mesh

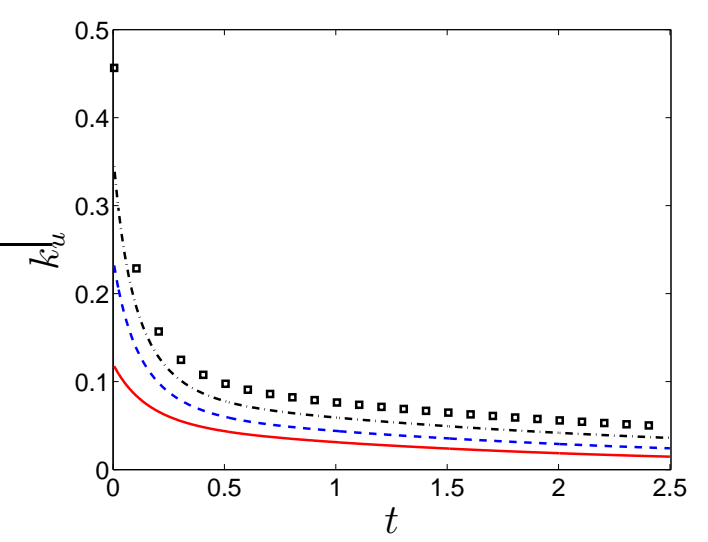

(b) $64 \times 64 \times 64$ mesh

Figure 4: DHIT using the LRN PANS model and the Dynamic Smagorinsky model. Modelled turbulence energy decaying with time. $-f_{k}=0.2 ;-.-: f_{k}=0.4 ;-. .: f_{k}=0.6$; $f_{k}=0.8$.

value of $f_{k}$ is, the smaller becomes the effect of the model on the resolved flow. With the current grid for the channel flow (Table 1), it was found 
that for $f_{k} \leq 0.3$, the model plays an insignificant role in the simulation, i.e. $\nu_{u} \simeq 0$. In the following discussion, only results computed with $f_{k}=0.4$, 0.5 and 1.0 are presented. Note that with $f_{k}=1.0$, both the standard and the LRN PANS models should return to their respective base models in the context of RANS modelling.

Figure 5 compares the mean streamwise velocities, computed using the standard PANS model and the proposed LRN PANS model with the DNS data by Hoyas and Jimenez (2008). For $f_{k}=1.0$, the computations give steady RANS solutions. The LRN PANS model returns to the AKN LRN $k-$ $\varepsilon$ model, while the standard PANS model becomes identical to the standard $k-\varepsilon$ model. This has indeed been reflected in the predictions, as illustrated in Fig. 5 for $f_{k}=1.0$. The poor prediction by the standard $k-\varepsilon$ model is not surprising, since the model has been integrated to the wall surface on a low Reynolds number grid.

The velocity profiles using $f_{k}=0.4$ and $f_{k}=0.5$ are also shown in Fig. 5 . The standard PANS model with $f_{k}=0.5$ has produced an overall erroneous velocity distribution from the viscous sublayer to the logarithmic layer, but the prediction in the central part is closer to the DNS data. The LRN PANS model is able to give a generally improved tendency of the profile, in spite of an over-prediction in the logarithmic layer. The improvement is particularly obvious in the viscous sublayer, due to the correct asymptotic properties 


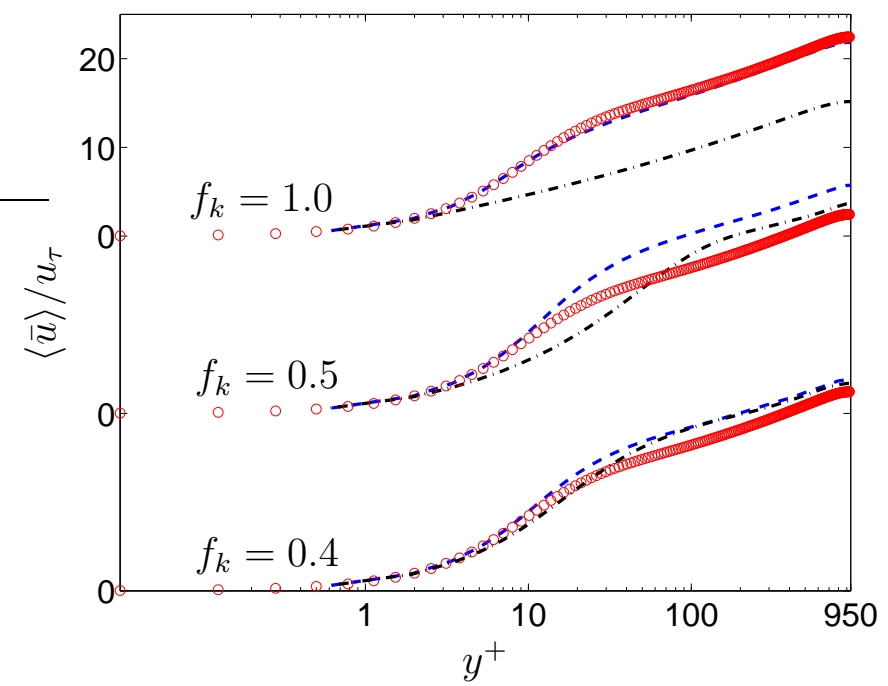

Figure 5: Turbulent channel flows at $R e_{\tau}=950$ : distributions of streamwise velocity. - _ - : LRN PANS model; -. . : standard PANS model; ॰: DNS data (Hoyas and Jimenez, 2008).

inherent in the LRN PANS formulation. Using $f_{k}=0.4$, the standard PANS and the LRN PANS models have produced very similar results that are in reasonable agreement with the DNS data.

As shown in Fig. 5, the results are fairly sensitive to the value of $f_{k}$. A value of $f_{k}=0.5$ gives worse agreement with DNS data than $f_{k}=0.4$. The reason is that the modelled turbulent viscosity, $\nu_{u}$, increases by a factor of about two when $f_{k}$ is increased from 0.4 to 0.5 . This is further illustrated in Fig. 6, where the modelled turbulent viscosity, $\nu_{u}$, is presented. As expected, $\nu_{u}$ increases for increasing $f_{k}$. It is noted here that a similar sensitivity to $f_{k}$ was also observed in the computation of hill flow. With the LRN PANS model, the turbulent viscosity reaches approximately 3.5 and 7 in the center 
of the channel for $f_{k}=0.4$ and $f_{k}=0.5$, respectively. The LRN PANS model gives very small values of $\left\langle\nu_{u}\right\rangle / \nu$ in the near-wall region (it is close to zero in the viscous sublayer for both $f_{k}=0.4$ and $f_{k}=0.5$, see Fig. $\left.6(\mathrm{~b})\right)$.

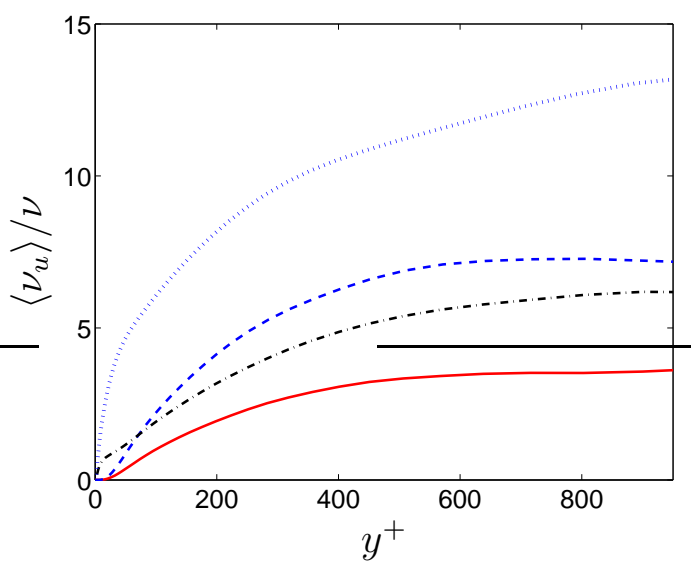

(a) $R e_{\tau}=950$

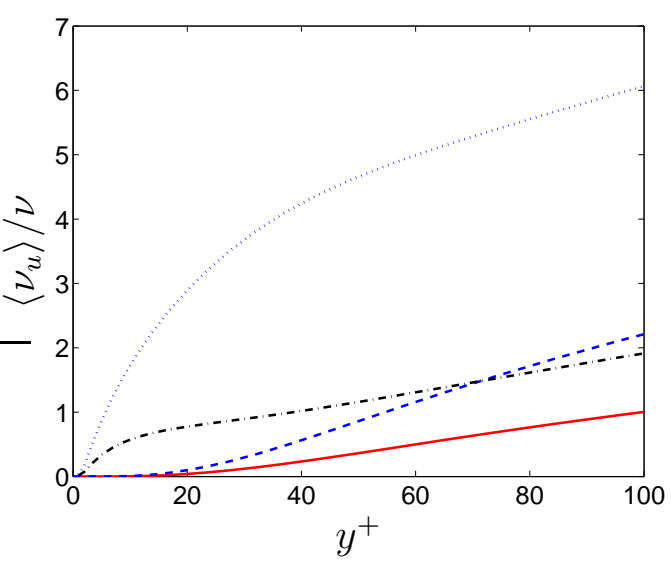

(b) $R e_{\tau}=950$, zoomed view

Figure 6: Modelled turbulent eddy viscosity, $\left\langle\nu_{u}\right\rangle / \nu$. LRN PANS, $f_{k}=0.4 ;---$ : LRN PANS, $f_{k}=0.5 ; \ldots$ : PANS, $f_{k}=0.4 ; \ldots \ldots$ : PANS, $f_{k}=0.5$.

Using $f_{k}=0.4$ gives the best predictions for both the standard and the LRN PANS models. To highlight the modelling performance for near-wall turbulence in channel flow computations, the results computed with $f_{k}=0.4$ are presented below for the resolved turbulence statistics. Note that, as shown in Table 1, the grid is fairly fine and it is suitable for wall-resolved LES. It is thus expected that the PANS formulation, with both the standard and the LRN models, should produce LES-comparable predictions.

Figure 7(a) compares the resolved turbulent normal stresses to DNS data. It is shown that the resolved turbulent fluctuations computed with the LRN 
PANS model are in reasonable agreement with DNS data. In general, the LRN model shows better performance than the standard model. This is particularly true in the vicinity of the wall surface, namely, over the viscous sublayer for $y^{+} \leq 10$. Figure $7(\mathrm{~b})$ shows $f_{k}$ computed from the predicted resolved and modelled turbulence kinetic energy. It is seen that, due to the LRN modifications, the predicted $f_{k}$ is much smaller than the prescribed one, mainly because $k_{u}$ in the near-wall viscous sublayer is dampened as it should.

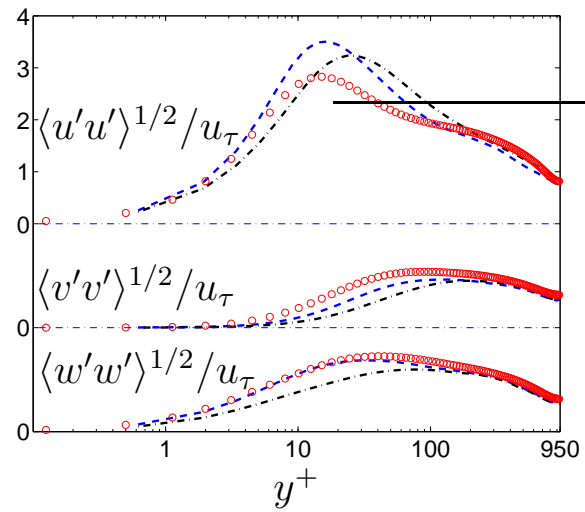

(a) Resolved turbulent fluctuations

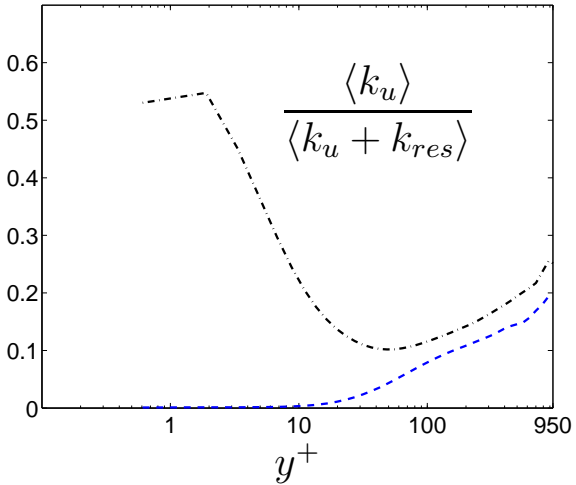

(b) Ratio of modelled to total turbulent kinetic energy. $k_{r e s}=0.5\left(u^{\prime} u^{\prime}+v^{\prime} v^{\prime}+w^{\prime} w^{\prime}\right)$.

Figure 7: Turbulent channel flow at $R e_{\tau}=950$ : Turbulent normal stresses with $f_{k}=0.4$. - - - : LRN PANS model; -.. : standard PANS model; o: DNS data (Hoyas and Jimenez, 2008).

Figure 8(a) shows the resolved Reynolds shear stress, $-\left\langle u^{\prime} v^{\prime}\right\rangle$, with $f_{k}=$ 0.4 in comparison with DNS data. The turbulent shear stress obtained with the standard PANS model in the viscous sublayer and up to $y^{+} \approx 50$ is 
smaller than that predicted with the LRN model. This is closely associated to large values of the modelled eddy viscosity and modelled shear stress (see Fig. 8(b)) by the standard PANS model in the near-wall region. The results, as illustrated in Figs. 7(a) and 8(a), show that the LRN formulation has indeed introduced better wall-limiting behavior into the modelling compared with the standard PANS model. It is also demonstrated that correct nearwall asymptotic modelling improves the predictions of the resolved turbulent Reynolds stresses and turbulent kinetic energy.

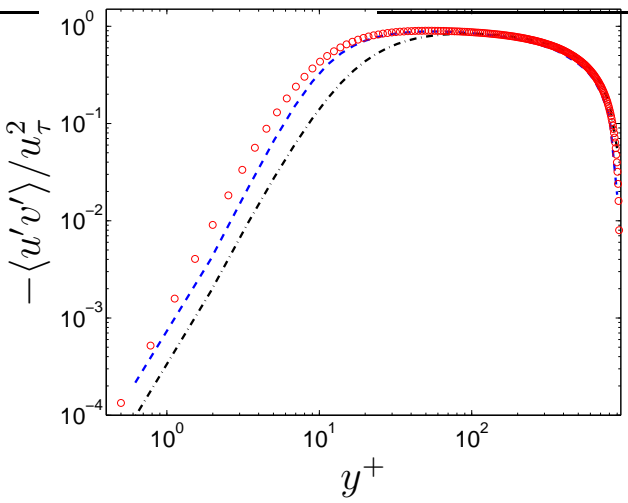

(a) Resolved stresses.

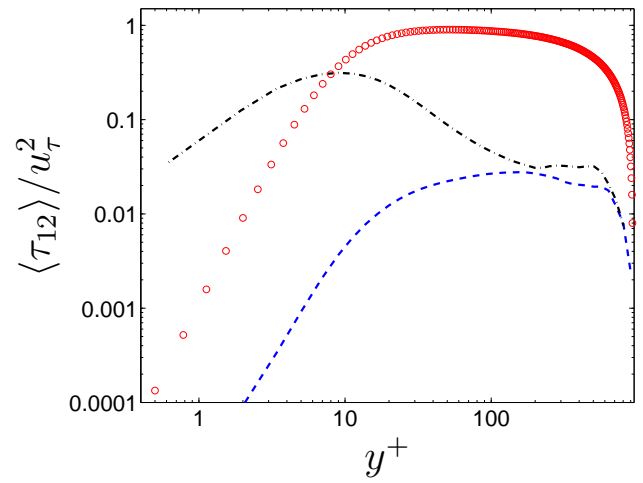

(b) Modelled stresses.

Figure 8: Turbulent channel flow at $R e_{\tau}=950$ : Turbulent shear stress with $f_{k}=0.4$. - . - : LRN PANS model; _.. . : standard PANS model; ○: DNS data (Hoyas and Jimenez, 2008).

The PANS modelling represents the effect of the unresolved part on the resolved turbulence. In both the standard and the LRN PANS models, $f_{k}$ (with $f_{\varepsilon} \equiv 1.0$ ) is the only parameter that differentiates the PANS formulations from their respective RANS base models. It is thus interesting to 
explore the effect of $f_{k}$ on the budget of the modelled turbulence transport equation. This is shown in Fig. 9 for the equation of the modelled turbulent kinetic energy, $k_{u}$, for channel flow at $R e_{\tau}=950$. Figures $9(\mathrm{a})$ and (b) present the $k_{u}$ budget for the standard PANS and the LRN PANS models for $f_{k}=1.0$ and the budget of $k$ for DNS data is shown in Fig. 9(c). As compared to the standard $k-\varepsilon$ model, the improvement achieved by the AKN LRN model is apparent in comparison with the DNS data. Very close to the wall surface, both the dissipation term and the molecular diffusion term have been over-estimated with the standard PANS model in Fig. 9(a) and under-predicted by the LRN PANS model in Fig. 9(b). This may largely be attributed to the boundary condition of $\varepsilon_{u}$ on the wall surface, which has been approximated in the computations from the balance of the two terms in the vicinity of the wall surface.

As an example, the $k_{u}$ budgets with $f_{k}=0.4$, where both the standard and LRN PANS models have produced the best predictions, are plotted in Fig. 10. The values of budget terms are in the near-wall region much lower with the LRN PANS model than with the standard PANS, see Figs. 10(a) and (b). The reason to the small production term in Fig. 10(b) is the small turbulent viscosity, $\nu_{u}$, see Fig. 6(b) (it is very small for $y^{+} \leq 20$ ), which has been mainly caused by the damping functions, $f_{\mu}$ and $f_{2}$. The incorporation of $f_{2}$ into the sink term in the $\varepsilon_{u}$ equation has modified the $C_{\varepsilon 2}^{*}$ coefficient, 


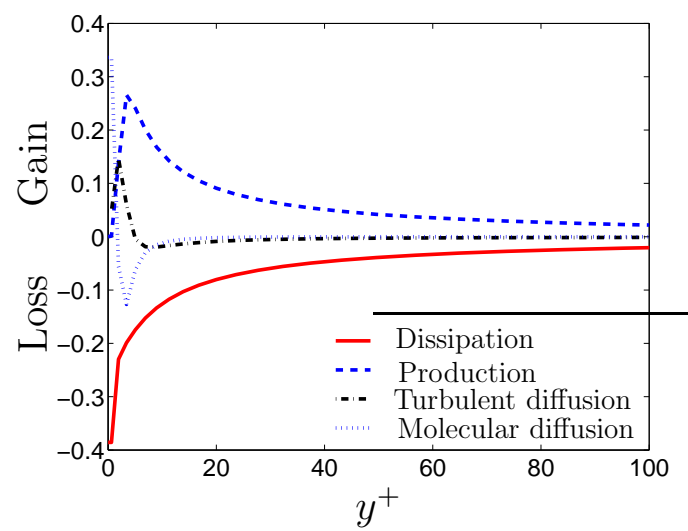

(a) Standard PANS

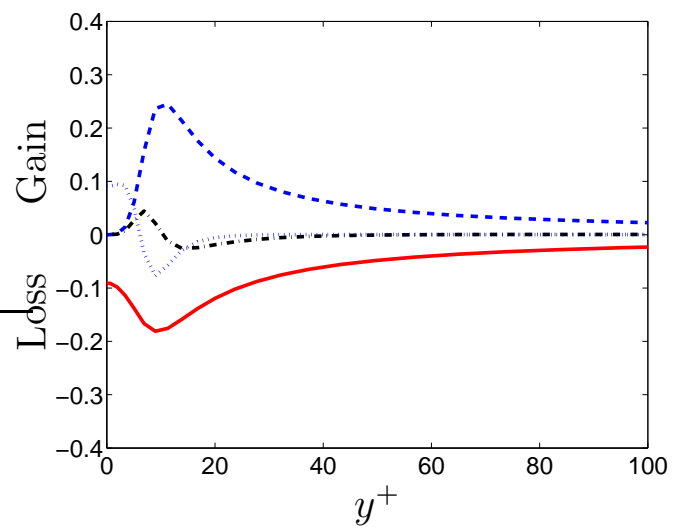

(b) LRN PANS

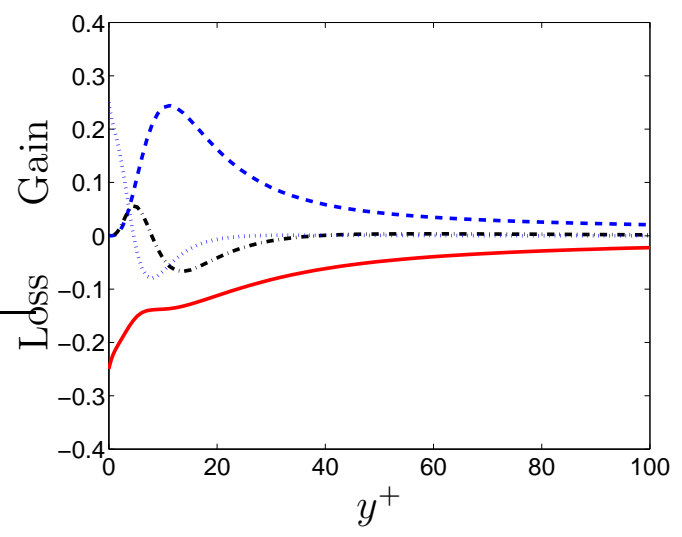

(c) DNS

Figure 9: Turbulent channel flows at $R e_{\tau}=950$ : Budget for the $k_{u}$ equation with $f_{k}=1.0$.

see Eq. 16, rendering large values of $\varepsilon_{u}$ and hence small $k_{u}$ and $\nu_{u}$. Because of the small $\nu_{u}$, the production with the LRN PANS model is significantly reduced in this region, whereas it reaches its maximum with the standard PANS model. As a consequence, the locations of the peak values for both the production and the dissipation terms have with the LRN PANS model moved out of the buffer region. The reduction in the production of the LRN 
PANS model has entailed reduced viscous and turbulent diffusion terms. Nevertheless, in the outer region, the budgets of the standard PANS and LRN PANS are very similar, see Figs. 10(a) and 10(d). Here, both models have indicated a balance between the modelled production and dissipation, implying that local equilibrium holds approximately in both PANS models.

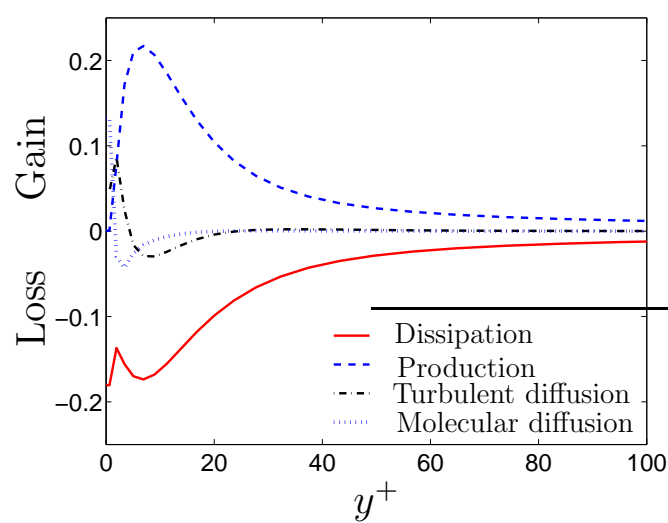

(a) Standard PANS, near-wall

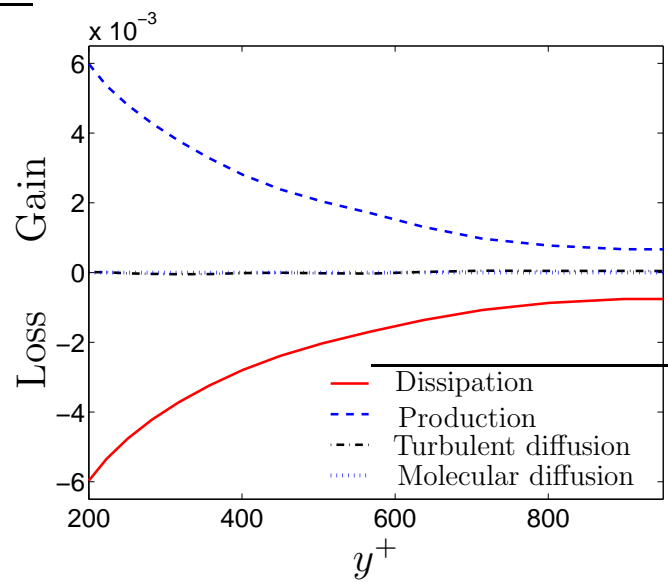

(c) Standard PANS, $y^{+} \geq 200$

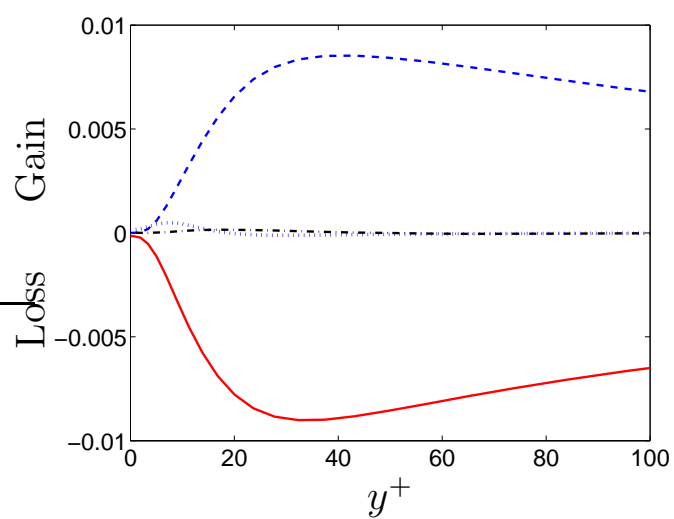

(b) LRN PANS, near-wall

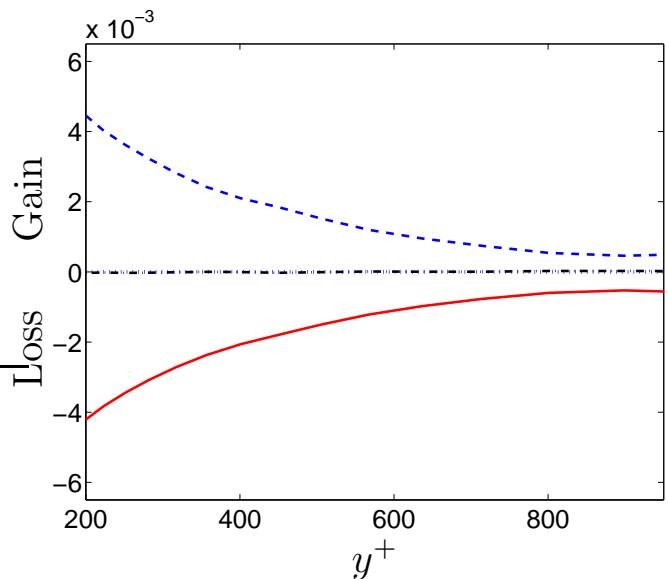

(d) LRN PANS, $y^{+} \geq 200$

Figure 10: Turbulent channel flows at $R e_{\tau}=950$ : Budget for the $k_{u}$ equation with $f_{k}=0.4$. 
We have so far explored in channel flow simulations the performance of the proposed LRN PANS model in comparison with the standard PANS model. With the present grid resolution (see Table 1), both PANS models have actually enabled LES-like performance. It is desired that the PANS modelling in the simulation should present turbulence-resolving capabilities equivalent to SGS modelling in LES. It is then interesting to compare the LRN PANS model with one of the best available SGS models, namely, the Dynamic Smagorinsky model. Figure 11 presents a comparison of the time-averaged streamwise velocity and the resolved velocity fluctuations obtained, respectively, with the Dynamic Smagorinsky model and the LRN PANS model. As seen, the LRN PANS (with $f_{k}=0.4$ ) gives slightly better agreement with DNS data than the Dynamic SGS model. This suggests that the proposed LRN PANS model has indeed enabled LES-like performance on an LES grid. It should be mentioned, moreover, that the required CPU time is actually somewhat lower for the LRN PANS model than for the dynamic model that has invoked additional test-filtering operations to compute the model coefficient.

\subsection{Periodic hill flow $(R e=10600)$}

The periodic hill flow was computed to verify PANS performance in modelling turbulent flow separation and reattachment. For this test case, the simulation is compared with an accurate wall-resolved LES (Fröhlich et al., 


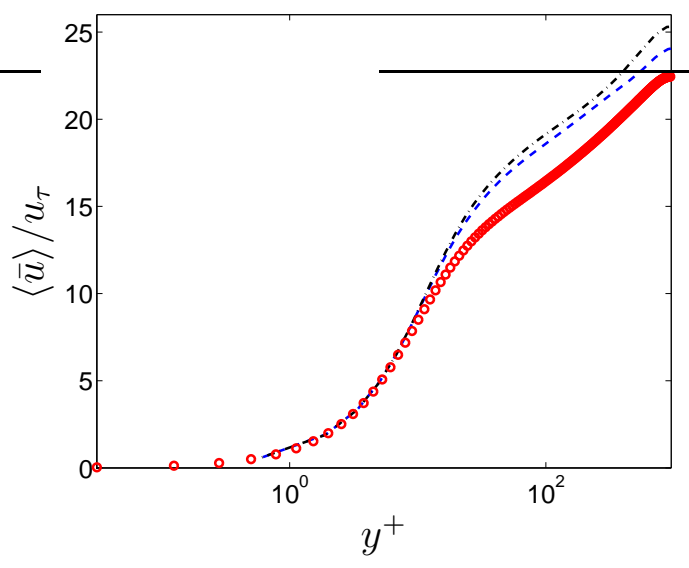

(a) Mean velocity

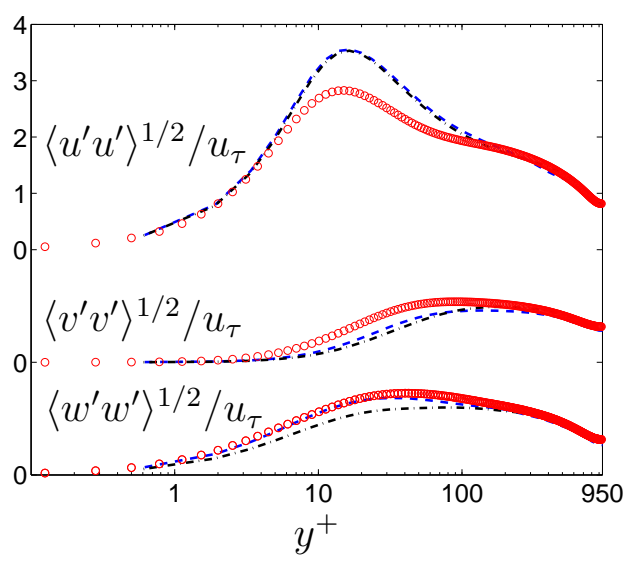

(b) Reynolds stresses

Figure 11: Turbulent channel flow at $R e_{\tau}=950$ : mean velocity and resolved turbulent velocity fluctuations. - - - : LRN PANS model; - - - : Dynamic Smagorinsky model; o: DNS data (Hoyas and Jimenez, 2008).

2005). It is noted here that the present grid, $160 \times 80 \times 32$ cells in a domain of $9 h \times 3.035 h \times 4.5 h$, is much coarser than the wall-resolved LES mesh using $196 \times 128 \times 186$ cells in a domain of $9 h \times 3.035 h \times 9 h$. The mean flow and the resolved turbulence statistics presented below were obtained by time-averaging and spatial-averaging over the spanwise direction.

With the same mesh, a number of computations were made using various values of $f_{k}$, with $f_{k}=0.4,0.5,0.6,0.8$ and 1.0 , in order to explore the effect of $f_{k}$. In comparison with the LES result, it was found that the PANS computation with $f_{k}=0.4$, using either the standard or the LRN model, produced the best prediction of the separation bubble on the lee side of the hill in terms of the locations of both the separation and the reattachment. The 

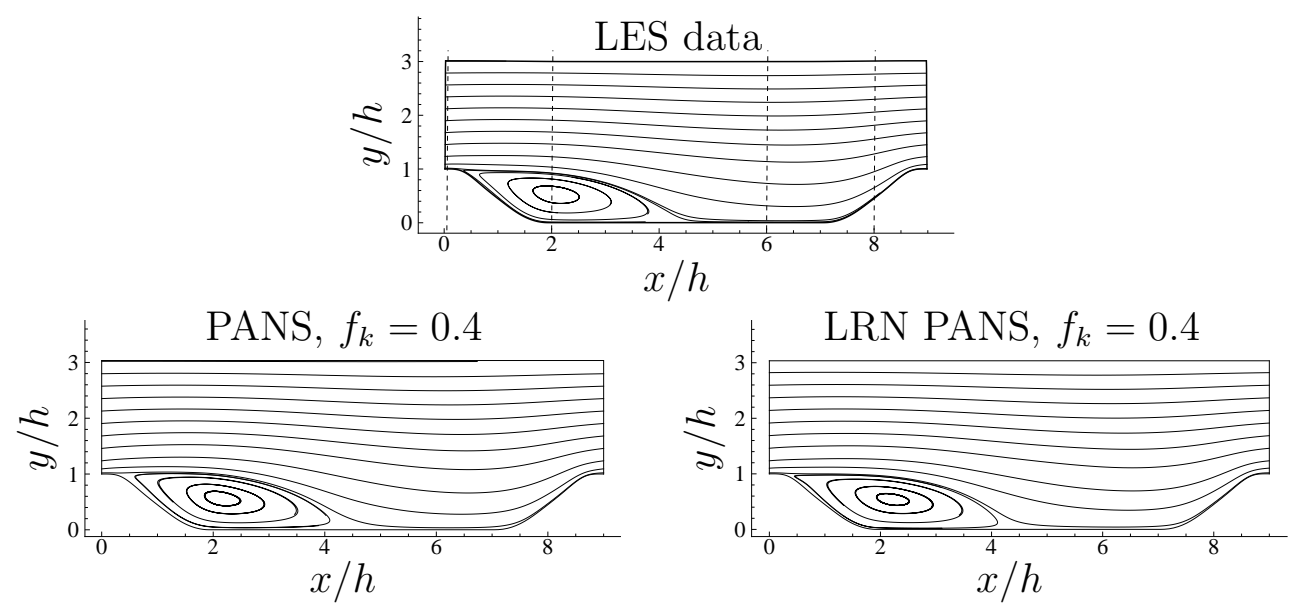

Figure 12: Turbulent periodic hill flow, $R e=10600$ : streamlines of time-averaged flow from PANS computations in comparison with wall-resolved LES data (Fröhlich et al., 2005).

streamlines of the time-averaged flow from PANS computations are shown in Fig. 12, in comparison with the LES solution (Fröhlich et al., 2005). The LES result indicates that the flow is separated at $x_{s}=0.2 h$ shortly after the hill crest and is reattached at $x_{r}=4.7 \mathrm{~h}$ after the hill foot. The recirculation bubble extends over almost $50 \%$ of the domain in the streamwise direction. As shown, both models produced reasonable predictions of the mean flow.

The locations of flow separation and reattachment, $x_{s}$ and $x_{r}$, respectively, are plotted in Fig. 13 (a) and (b) as function of $f_{k}$. As seen, the LRN PANS model produces accurate predictions of $x_{s}$ and $x_{r}$ with $f_{k}=0.4$, whereas the standard PANS model shows a somewhat earlier flow separation and the reattachment is slightly delayed. With increasing values of $f_{k}$, the location 


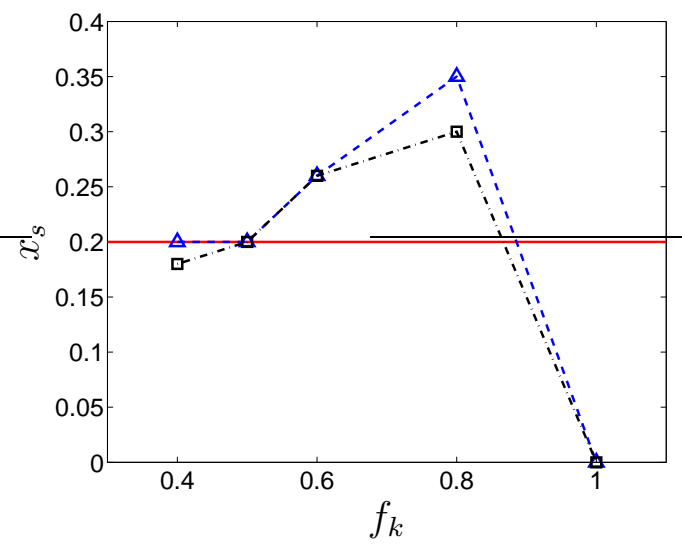

(a) Separated locations

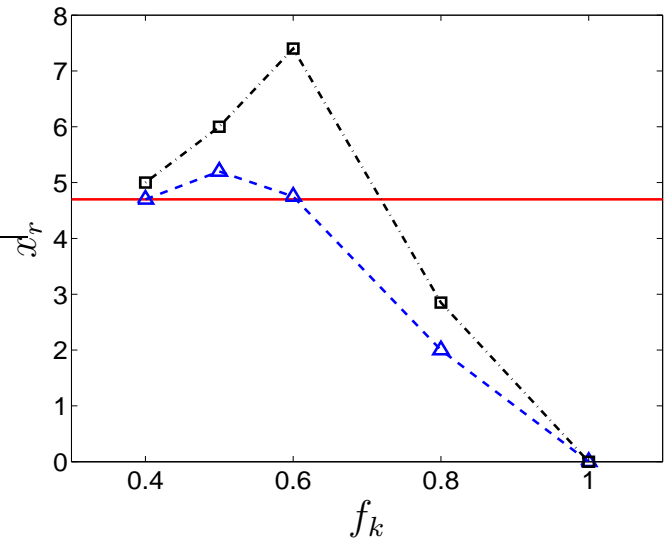

(b) Reattached locations

Figure 13: Periodic hill flow, $R e=10600$ : Locations of separation and reattachment, $x_{s}$ and $x_{r}$, versus parameter $f_{k}$ used in PANS computations. Standard PANS gives $x_{s} \approx 0.18$ and $x_{r} \approx 5.0$, and LRN PANS gives $x_{s} \approx 0.2$ and $x_{r} \approx 4.7$ with $f_{k}=0.4$, compared to the LES data $x_{s}^{L E S}=0.2$ and $x_{r}^{L E S}=4.7 . \triangle:$ LRN PANS; $\square:$ standard PANS; — : LES data.

of flow separation is shifted downwards along the lee-side of the hill, and the reattachment location is first delayed and then moves back toward the hill foot. With $f_{k}=1.0$, both models produce incorrectly overall attached flow over the hill and between the hills, namely, $x_{s}=x_{r}=0$, as shown in Fig. 13. It should be noted that, for $f_{k}=1.0$, both models return to their respective RANS base models, giving steady solutions based on the standard $k-\varepsilon$ model and the AKN LRN $k-\varepsilon$ model.

We present below only the computations obtained with $f_{k}=0.4$, for which both the standard and the LRN models have produced the best pre- 
dictions. Figure 14(a) displays the $y^{+}$values of the wall-adjacent nodes and the friction coefficient along the bottom wall. Most of the wall-adjacent nodes are located at $y^{+} \approx 1.0$ or below, except for some points around the hill crest having relatively large values of $y^{+}$but, in general, within $y^{+}<3$. The value of $y^{+}$increases along the windward side of the hill due to an increase in the wall shear stress. The friction coefficient distributions on the lower wall are presented in Fig.14(b). The reverse flow in the separation bubble on the lee side of the hill is shown with negative values of $C_{f}$. Owing to a slightly more extended separation bubble, the standard model produces a somewhat more extensive reverse flow. Downstream of the reattachment, the recovery of the boundary layer flow is deterred by the presence of the next hill, where the friction coefficient shifts toward zero at the foot and then rises rapidly on the windward hill side due to flow acceleration.

To observe the capability of PANS modelling in resolving turbulent flows, the distributions of the mean flow and resolved turbulence statistics are compared in the following. The profiles were extracted at locations of $x=0.05 h$, $2.0 h, 6.0 h$ and $8.0 h$, respectively. The mean velocity components, $\langle\bar{u}\rangle$ and $\langle\bar{v}\rangle$, in the streamwise and vertical directions, respectively, as well as the resolved turbulent Reynolds stresses are compared with the LES data by Fröhlich et al. (2005). The first position, $x=0.05 h$, is located immediately after the hill crest, which may help to highlight any possible influence of the 


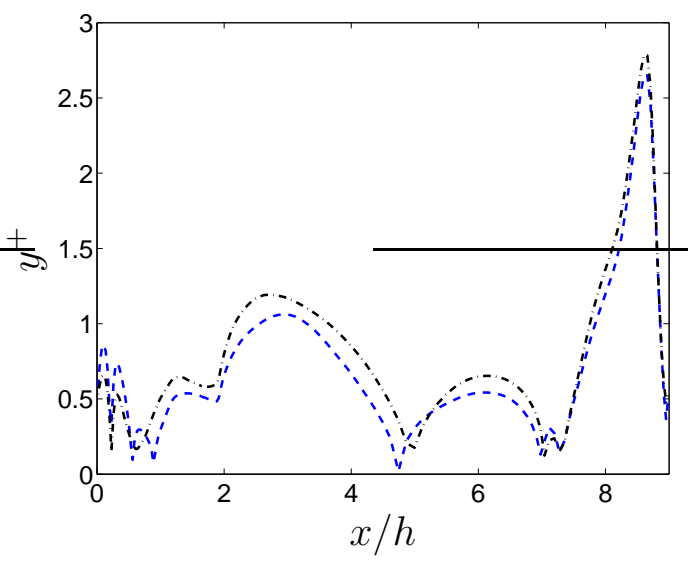

(a) Near-wall grid spacing

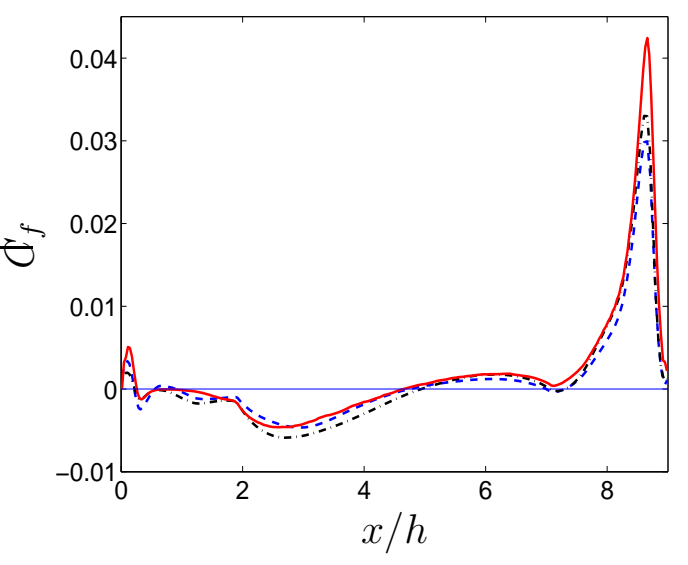

(b) Friction coefficient

Figure 14: Periodic hill flow, $R e=10600$ : Near-wall grid spacing and friction coefficient along the bottom wall. - - - : LRN PANS model; _... : standard PANS model; — — Wall-resolved LES data.

upstream flow prior to the separation onset. The second location, $x=2.0 \mathrm{~h}$, in the center of the recirculation bubble, is particularly interesting, since this position includes a free shear layer above the bubble, a reverse flow over the lower wall and a boundary layer on the top wall. After the reattachment, the profile at $x=6.0 h$ may highlight the flow recovery in the channel before reaching the windward side of the next hill. At $x=8.0 h$, the flow accelerates due to the contraction on the windward portion of the hill.

The mean velocity profiles are presented in Fig. 15 in comparison with the LES data. It is shown that both the standard and the LRN PANS models have produced very reasonable predictions, but some relatively large discrepancies are observed in the prediction by the standard model. Near 


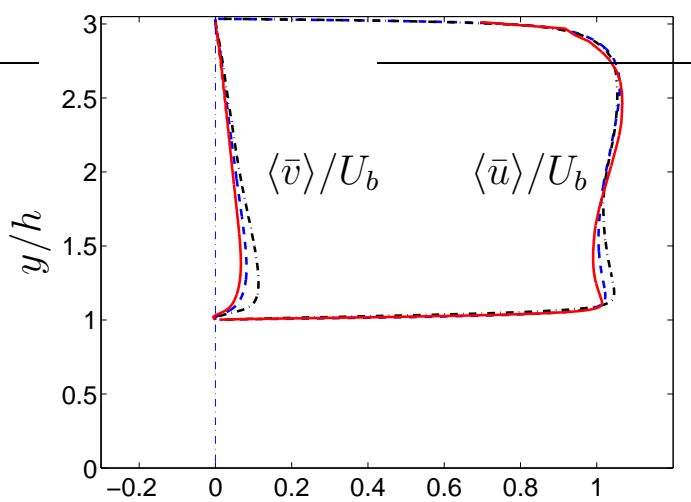

(a) $x / h=0.05$

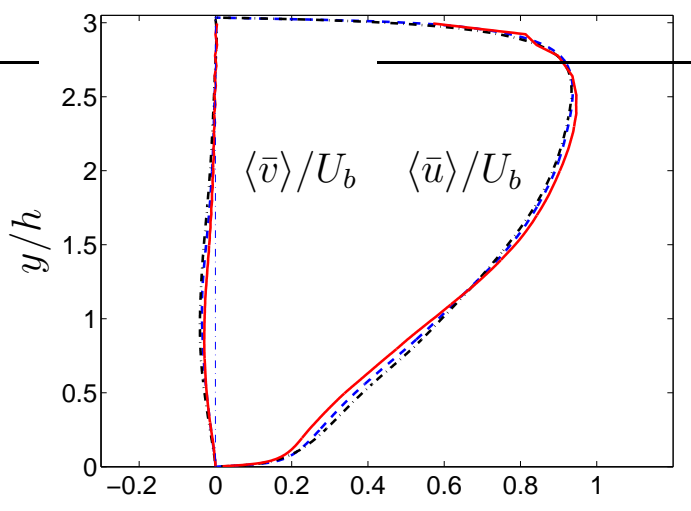

(c) $x / h=6.0$

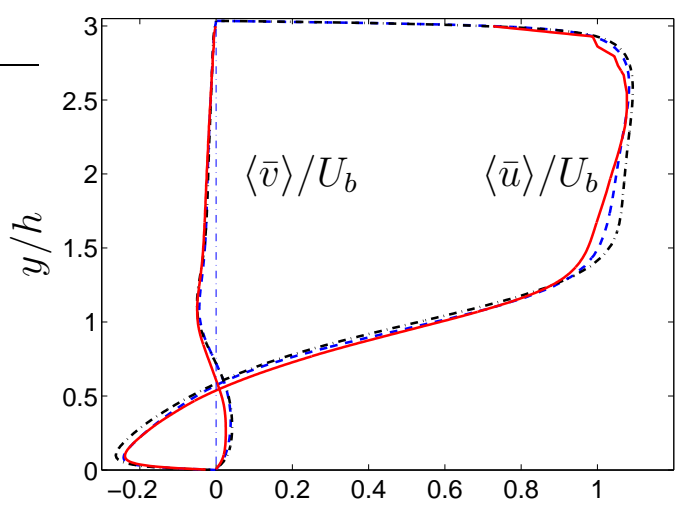

(b) $x / h=2.0$

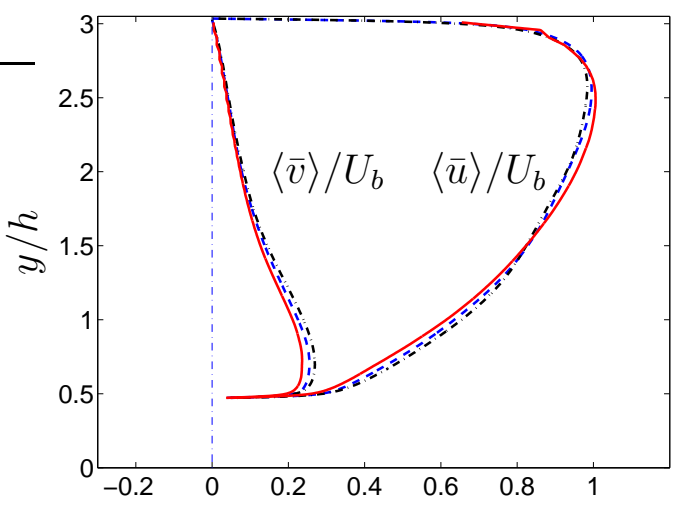

(d) $x / h=8.0$

Figure 15: Periodic hill flow, $\operatorname{Re}=10,600$ : Comparison of mean velocities. - - - : LRN PANS model; ... : standard PANS model; _ _ : Wall-resolved LES data.

the lower wall, as well as in the free shear layer (at $x=2.0 h$ ), the standard PANS has somewhat over-predicted the magnitude of $\langle\bar{u}\rangle$. The results are consistent to the $C_{f}$ distribution shown in Fig. 14(b).

Figure 16 presents the distributions of the resolved Reynolds stresses in comparison with the LES data. It is shown that, at $x=0.05 h$ before 
the separation occurs, the proposed LRN PANS model gives good prediction for the streamwise velocity fluctuations near the wall (the same for the spanwise velocity fluctuations, not shown here), whereas the standard model over-predicts the near-wall peaks of $\left\langle u^{\prime} u^{\prime}\right\rangle$. In the recirculation region (at $x=2.0 h)$, the resolved streamwise fluctuation is over-estimated around the peak at $y / h \approx 1$, especially by the standard PANS model. The LRN model presents better predictions of $\left\langle u^{\prime} u^{\prime}\right\rangle$ over the recirculation bubble at $x=2.0 h$. After the reattachment of the separation bubble, at $x=6.0 h$ and $x=8.0 h$, the LRN model produces generally better or similar predictions, as compared to the standard PANS model. For $\left\langle u^{\prime} u^{\prime}\right\rangle$, the proposed LRN model has shown improved turbulence-resolving capabilities in the attached boundary layer near the top and bottom walls and even in the near-wall reverse flow of the recirculation region (e.g. at $x=2.0 h$ ). For the resolved turbulent shear stress, $\left\langle u^{\prime} v^{\prime}\right\rangle$, the improvement over the standard PANS model in the predictions by the LRN model is also promising, particularly in the recirculation region and in the free shear layer. Near the top wall, $\left\langle u^{\prime} v^{\prime}\right\rangle$ is under-predicted. Although not shown here, it is indicated that, when the modelled part is included, the total turbulent shear stress in the wall layer agrees much better with the LES data.

The time-averaged PANS-modelled turbulent viscosities are shown in Fig. 17. Both models show very similar tendencies of change in the distribu- 


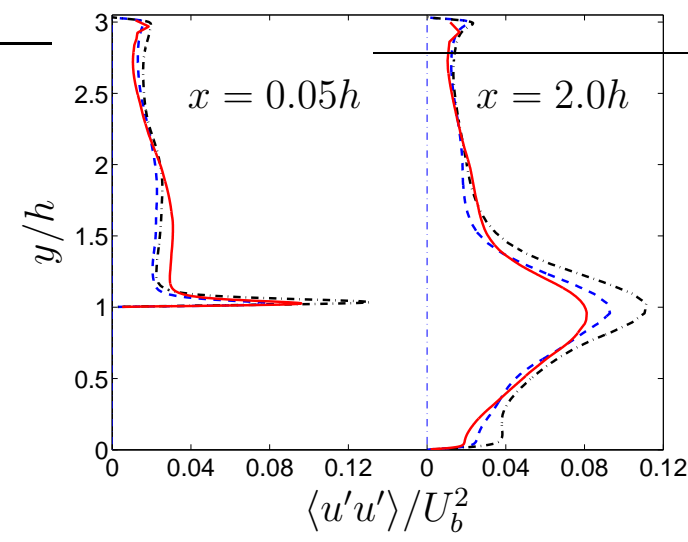

(a) $x / h=0.05$ and 2.0

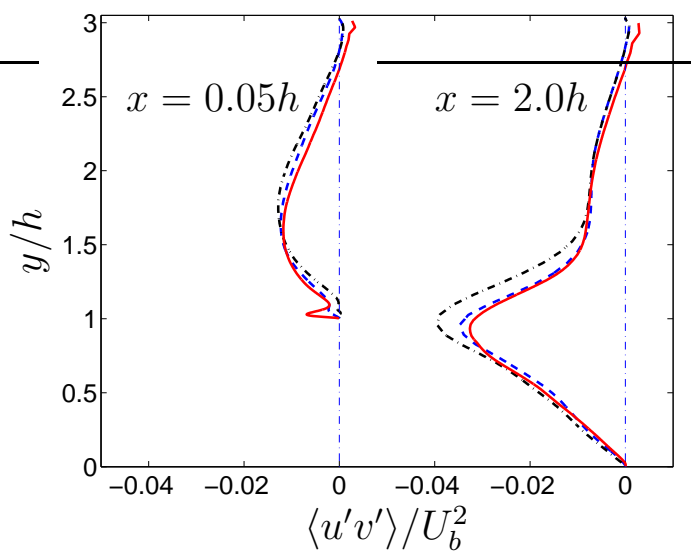

(c) $x / h=0.05$ and 2.0

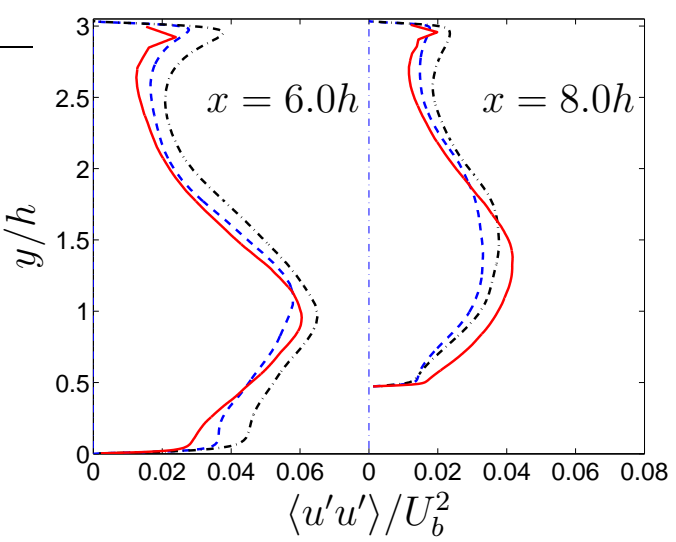

(b) $x / h=6.0$ and 8.0

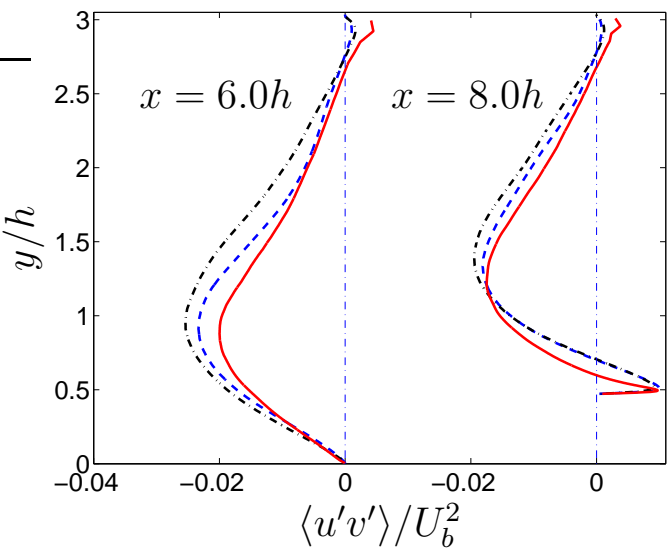

(d) $x / h=6.0$ and 8.0

Figure 16: Periodic hill flow, $R e=10600$ : Profiles of resolved Reynolds stresses. - - - : LRN PANS model; _. - : standard PANS model; — — : Wall-resolved LES data.

tions of $\nu_{u}$. Nonetheless, the standard PANS model produces larger values of eddy viscosities than the LRN PANS model, in general by more than $40 \%$ in off-wall regions. This is similar to that observed in turbulent channel flow computations (see Fig.6), as a consequence of the LRN formulation. 


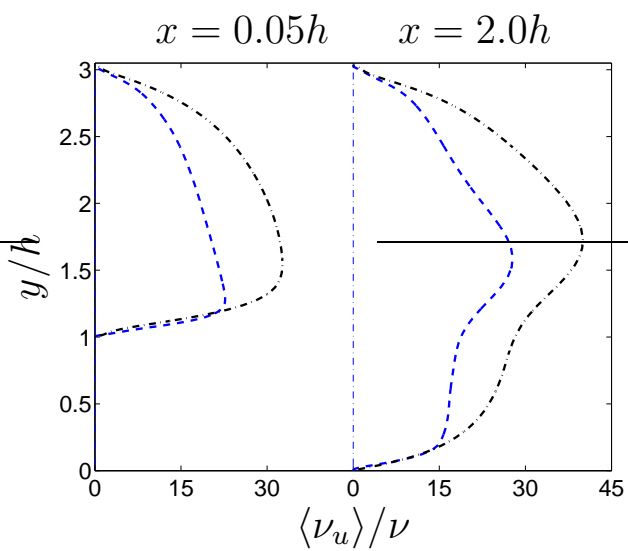

(a) $x / h=0.05$ and 2.0

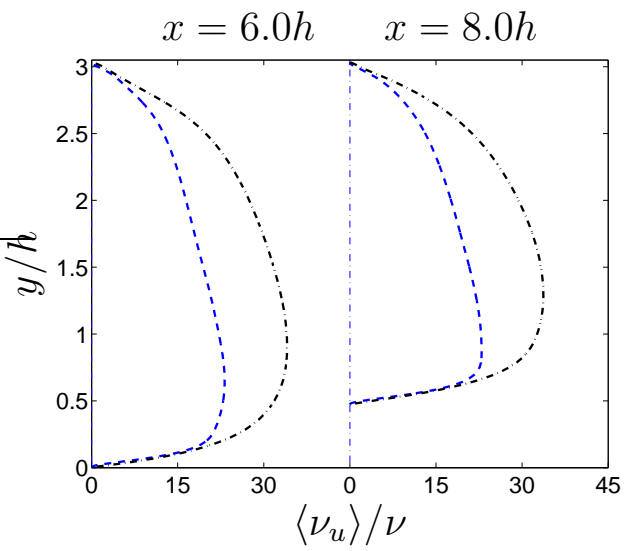

(b) $x / h=6.0$ and 8.0

Figure 17: Periodic hill flow, $R e=10$ 600: PANS-modelled turbulent viscosity. - - - : LRN PANS model; -... : Standard PANS model.

\subsection{Effect of turbulent Prandtl numbers in the LRN PANS model}

One of the major purposes with the development of PANS modelling was to enable the model to produce turbulence-resolving predictions, as a modelling approach compromised between RANS and LES. There have been other models developed for similar purposes, such as the PITM (Schiestel and Dejoan, 2005). In addition to modifying the sink term in the $\varepsilon_{u}$ equation, the PANS modelling has further re-formulated the turbulent Prandtl numbers, $\sigma_{k u}$ and $\sigma_{\varepsilon u}$, in the resulting $k_{u}$ and $\varepsilon_{u}$ equations, respectively, as given in Eqs. 14, 10 and 17. In order to investigate the influence of the turbulent Prandtl numbers in PANS modelling, we have carried out simulations with the LRN PANS model without modifying $\sigma_{k u}$ and $\sigma_{\varepsilon u}$, taking the values from the LRN RANS base model by setting $\sigma_{k u}=\sigma_{k}$ and $\sigma_{\varepsilon u}=\sigma_{\varepsilon}$. We denote 
this model LRN PANS $-\sigma$. In both LRN PANS models we have set $f_{\varepsilon}=1.0$ and $f_{k}=0.4$, which means that the modelled turbulent diffusion for $k_{u}$ and $\varepsilon_{u}$ in the LRN PANS model is larger than that in the LRN PANS $-\sigma$ model by a factor of $f_{k}^{-2} \simeq 6$.

For channel flow using the grid resolution in Table 1, the influence of $\sigma_{k u}$ and $\sigma_{\varepsilon u}$ is shown in Fig. 18. The LRN PANS $-\sigma$ model has produced a turbulent viscosity that is about $35 \%$ larger than the LRN PANS model, resulting in a slightly larger mean velocity in the buffer layer and in the logarithmic region. The increased turbulent viscosity by the LRN PANS $-\sigma$ model has been caused by its reduced turbulent diffusion for both $k_{u}$ and $\varepsilon_{u}$ as compared to the LRN PANS model. Although not shown, it is indicated here that the resolved Reynolds stresses by the two models are almost identical. The predicted velocity profile with no model, i.e. by DNS on a coarse grid, has also been included for comparison. As shown in Fig. 18(a), the result obtained with no model is surprisingly good, in some respects actually better than those with the PANS models and with the Dynamic Smagorinsky model in Fig. 11(a). However, a close inspection of the velocity profile reveals that, with no model, the slope is incorrect in the logarithmic layer. Similar results were also found in Davidson (2009) where, on the same mesh, simulation with no model (i.e. DNS) gave very good agreement with DNS data at $R e_{\tau}=500$. For the computation of hill flow at $R e=10600$, Fig. 19 presents a 


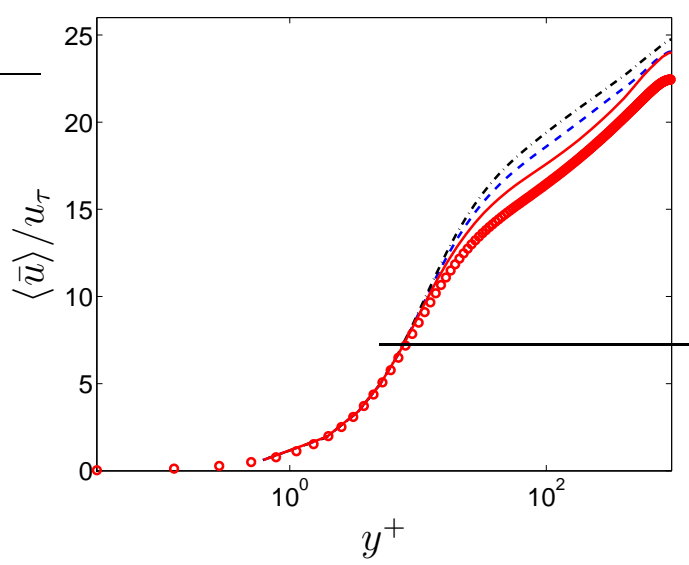

(a) Mean velocity

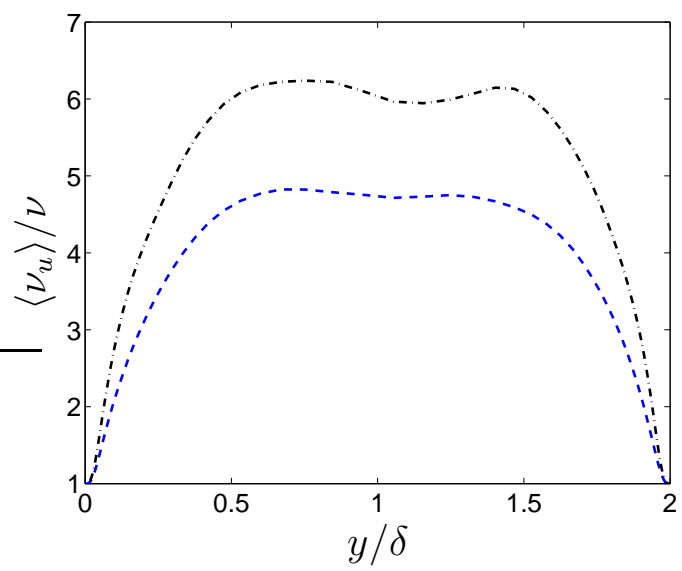

(b) Turbulent viscosity

Figure 18: Turbulent channel flow at $R e_{\tau}=950$ : mean velocity and turbulent viscosity. - - - : LRN PANS model; - . - : LRN PANS- $\sigma$ model; __ : Coarse DNS (no model); ○: DNS data (Hoyas and Jimenez, 2008).

comparison of the velocity profiles predicted by the Dynamic Smagorinsky model and by the LRN PANS $-\sigma$ model. Both models have produced similar results that are in good agreement with wall-resolved LES. Referring to Fig. 15, moreover, the velocity profiles obtained with the LRN PANS $-\sigma$ model are very similar to those obtained with the LRN PANS model. However, when comparing the resolved Reynolds stresses in Figs. 16 and 20, the resolved stresses predicted with the LRN PANS $-\sigma$ model are much smaller than with the LRN PANS model. This is due to the fact that the modelled turbulent viscosity is much larger with the LRN PANS $-\sigma$ model than with the LRN PANS model, as illustrated respectively in Figs. 21 and 17. This can be attributed to the large value of modelled turbulent diffusion in 


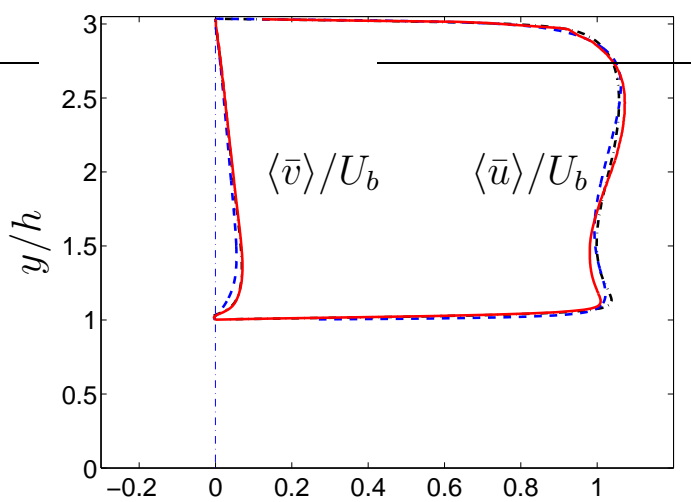

(a) $x / h=0.05$

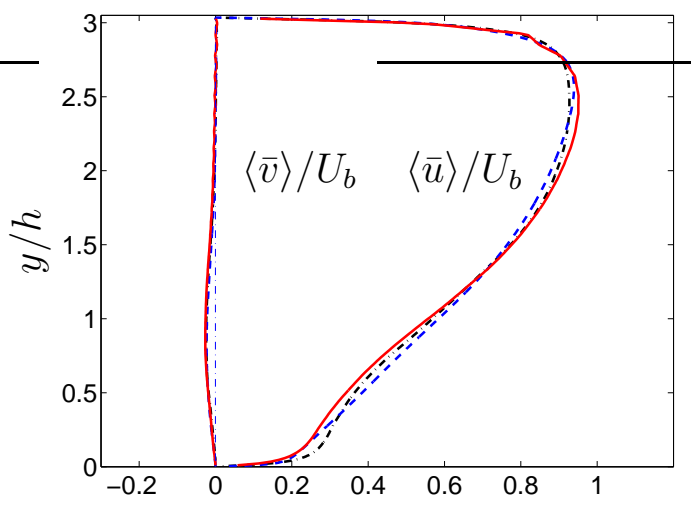

(c) $x / h=6.0$

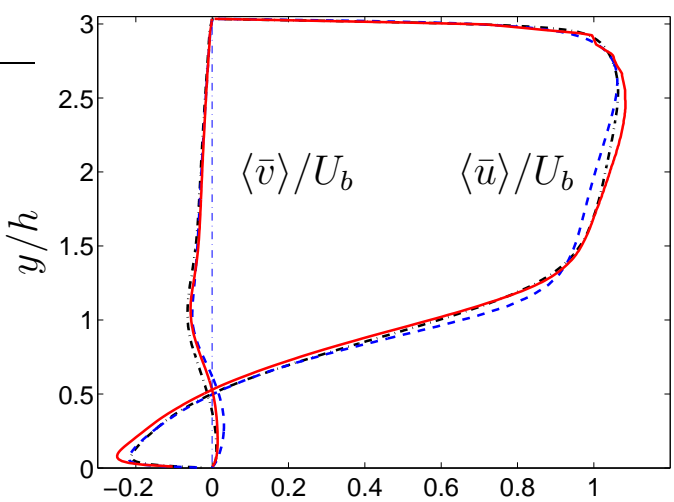

(b) $x / h=2.0$

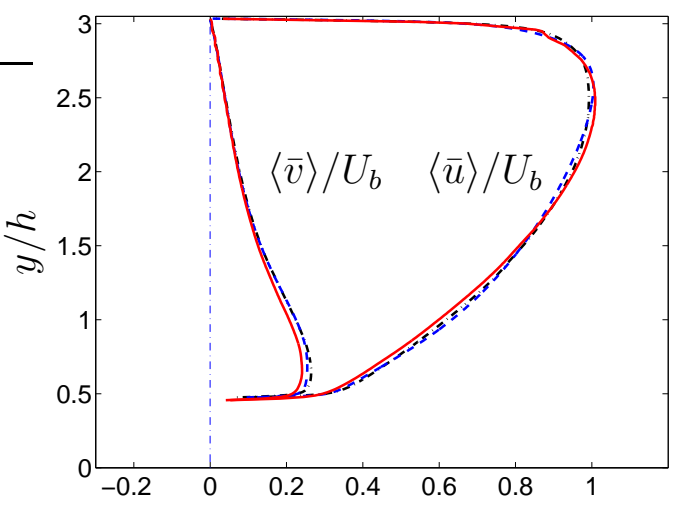

(d) $x / h=8.0$

Figure 19: Periodic hill flow, $R e=10600$ : Comparison of mean velocities. - - - : LRN PANS - $\sigma$ model; - . - : Dynamic Smagorinsky model; _ _ : Wall-resolved LES data.

the LRN PANS model, which, as mentioned above and as compared to the LRN PANS $-\sigma$ model, have induced more extensive diffusion of $k_{u}$ and $\varepsilon_{u}$. In the present hill flow the largest values of $k_{u}$ and $\varepsilon_{u}$ occur in the shear layer on the lee-side of the hill. With the LRN PANS model these peaks are strongly smoothed by turbulent diffusion, whereas they prevail with the 
LRN PANS $-\sigma$ model. It was found that the peaks of both $k_{u}$ and $\varepsilon_{u}$ are approximately five times larger with the LRN PANS- $\sigma$ model compared to the LRN PANS model and, consequently, the peak turbulent viscosities are approximately five times larger than with the LRN PANS $-\sigma$ model (see Figs. 17 and 21). The Dynamic Smagorinsky model, on the other hand, has shown a rather different behavior in resolving turbulent fluctuations and shear stresses. On the hill crest $(x=0.05 h)$ and at the location where the separation bubble exists, the dynamic model under-estimates $\left\langle u^{\prime} u^{\prime}\right\rangle$, but gives very good predictions of the resolved turbulent shear stress, $\left\langle u^{\prime} v^{\prime}\right\rangle$, as shown in Fig. 20. The modelled SGS eddy viscosity given by the Dynamic Smagorinsky model is much smaller than the LRN PANS- $\sigma$ model (Fig.21), and is about a half of, or less than, that by the LRN PANS model (Fig.17).

The large turbulent viscosities obtained with the LRN PANS- $\sigma$ model give rise to large values of modelled turbulent stresses. Figure 22 presents the total shear stresses, namely, the sum of the resolved and modelled shear stresses. Comparing with Fig. 20, it can be seen that the modelled shear stress has made a significant contribution to the total shear stresses.

\subsection{Periodic hill flow $(R e=37000)$}

The LRN PANS $-\sigma$ model has also been applied to the same hill flow as above but with a higher Reynolds number of $R e=37000$. The predictions are compared with experiments in Rapp (2008) and Rapp and Manhart 


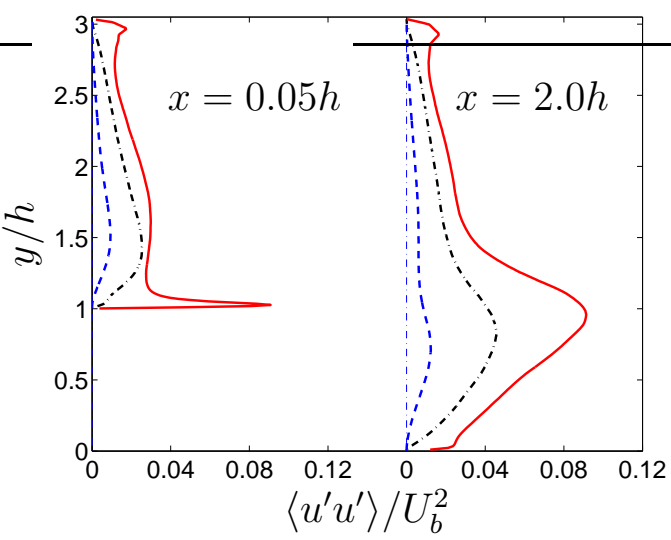

(a) $x / h=0.05$ and 2.0

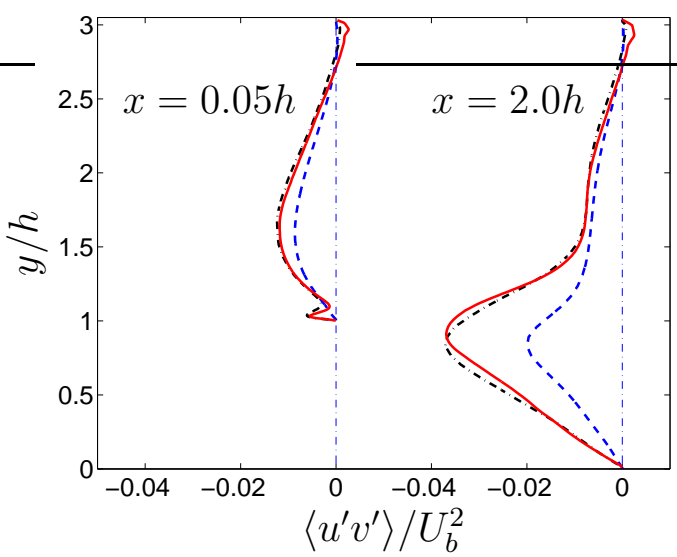

(c) $x / h=0.05$ and 2.0

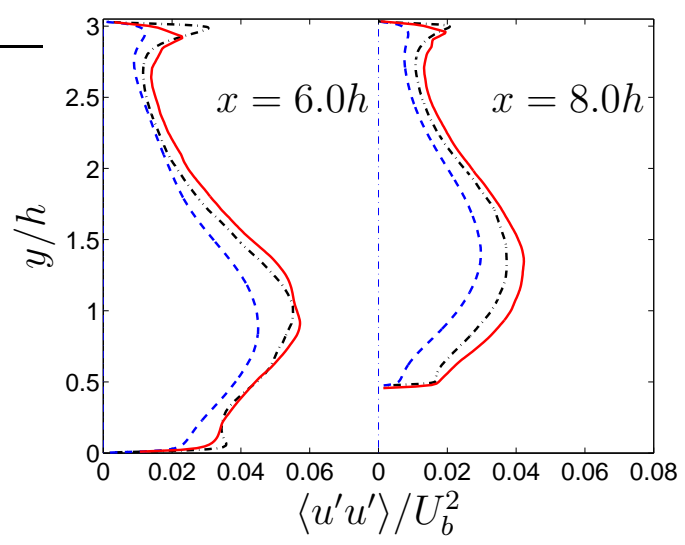

(b) $x / h=6.0$ and 8.0

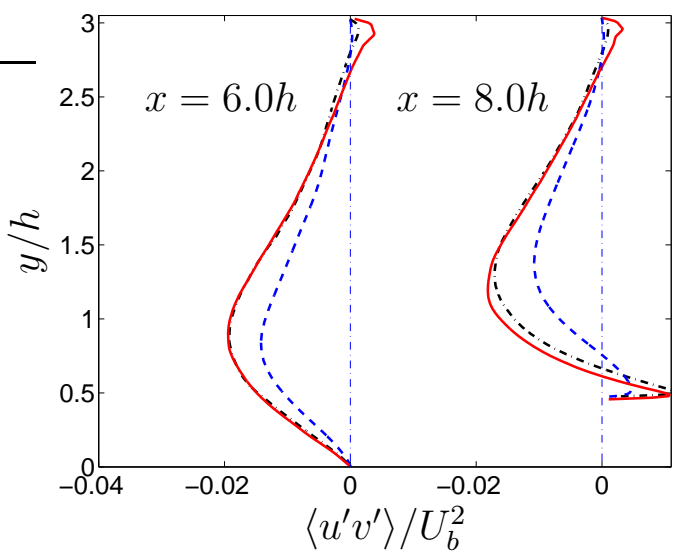

(d) $x / h=6.0$ and 8.0

Figure 20: Periodic hill flow, $R e=10600$ : Profiles of resolved Reynolds stresses. - - - : LRN PANS - $\sigma$ model; _. . : Dynamic Smagorinsky model; — : Wall-resolved LES data.

(2011), as well as with the computation using the Dynamic Smagorinsky model.

Figure 23 presents the mean velocity profiles. The mean streamwise velocity predicted by the LRN PANS $-\sigma$ model is in better agreement with 


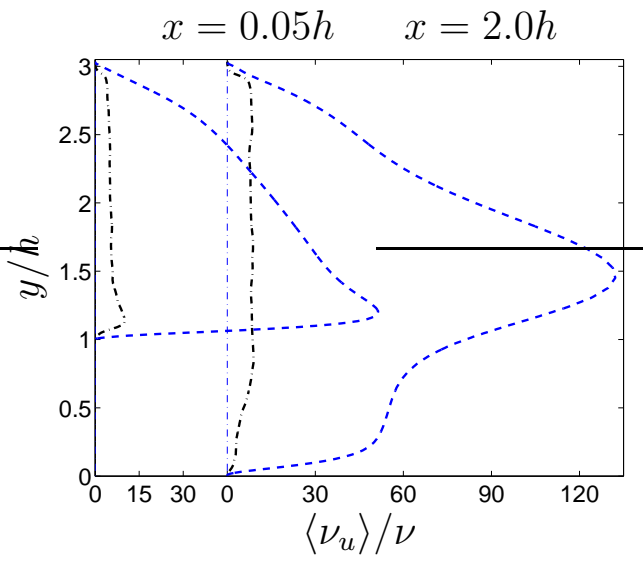

(a) $x / h=0.05$ and 2.0

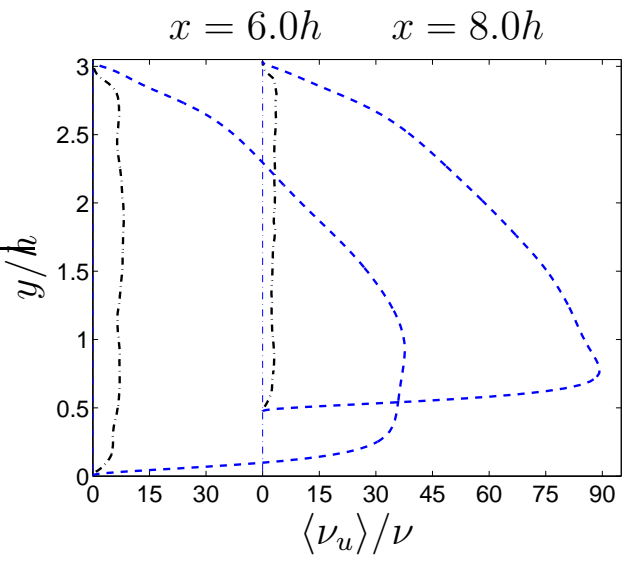

(b) $x / h=6.0$ and 8.0

Figure 21: Periodic hill flow, $R e=10600$ : Modelled turbulent viscosities. - - - : LRN PANS- $\sigma$ model. - - - : Dynamic Smagorinsky model.

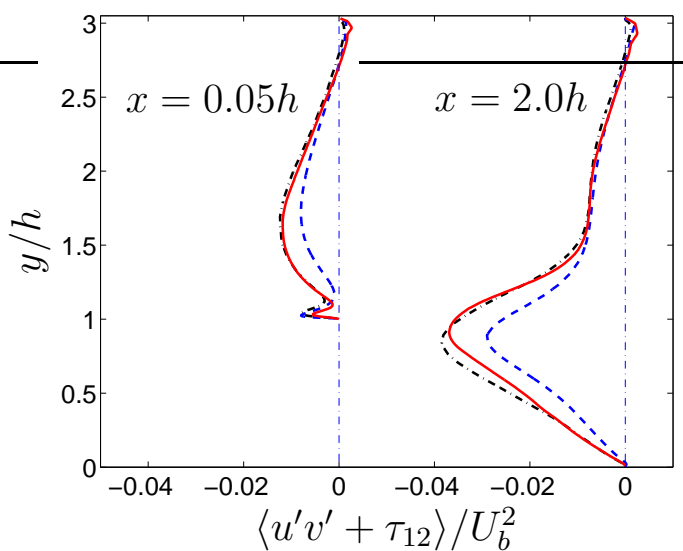

(a) $x / h=0.05$ and 2.0

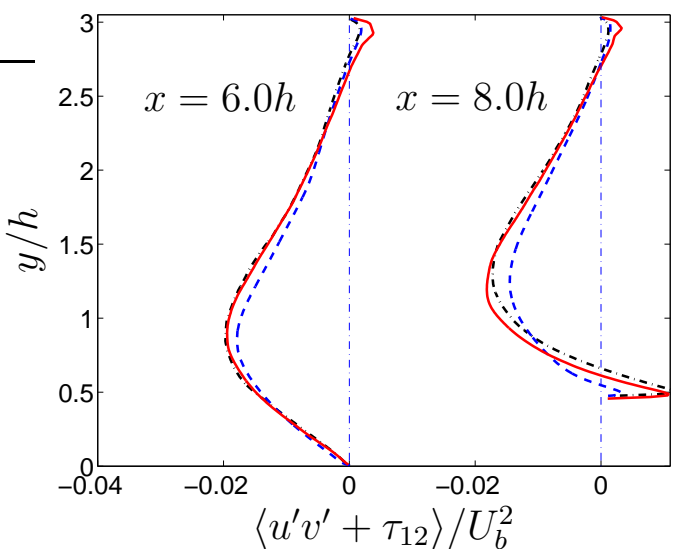

(b) $x / h=6.0$ and 8.0

Figure 22: Periodic hill flow, $R e=10600$ : Profiles of total (i.e. resolved and modelled) turbulent Reynolds shear stresses. : LRN PANS- $\sigma$ model; -.. : Dynamic Smagorinsky model; __ : Wall-resolved LES data.

the experimental data than the dynamic model. However, at the positions between $x / h=2$ and 6 (not shown), the LRN PANS $-\sigma$ model predicts 
a relatively extended separation bubble compared to both experiments and to the Dynamic Smagorinsky model. This has led to a somewhat slower flow recovery after the reattachment, as shown in Fig. 23(c). The Dynamic Smagorinsky model gives a somewhat more accurate prediction of the separation bubble, which may partly be explained by the fact that the model has under-predicted the near-wall velocity peak on the hill crest prior to the separation onset, see Fig. 23(a). This has caused a less intensive flow recirculation region, as indicated by the relatively weak reverse flow shown in Fig. 23(b). In general, the LRN PANS $-\sigma$ and the Dynamic Smagorinsky model predict the mean flow equally well. Although not shown, it is noted here that the LRN PANS- $\sigma$ model has produced somewhat closer agreement with the experimental data, as compared with the LRN PANS model for this test case.

The resolved turbulent shear stresses by the LRN PANS $-\sigma$ model are presented in Fig. 24 in comparison with the experimental data and with the prediction computed by the Dynamic Smagorinsky model. As shown, the results by both models are in reasonable agreement with experimental data, except at $x / h=2$ where the resolved shear stress has been sensibly underpredicted by the LRN PANS $-\sigma$ model. Similar to the results for $R e=10600$, due to large values of the modelled turbulent viscosity, the modelled shear stresses with the LRN PANS- $\sigma$ model has accounted for a larger part of 


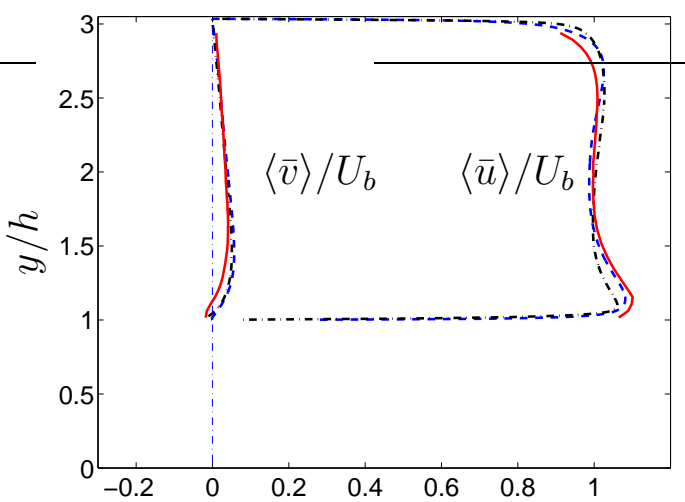

(a) $x / h=0.05$

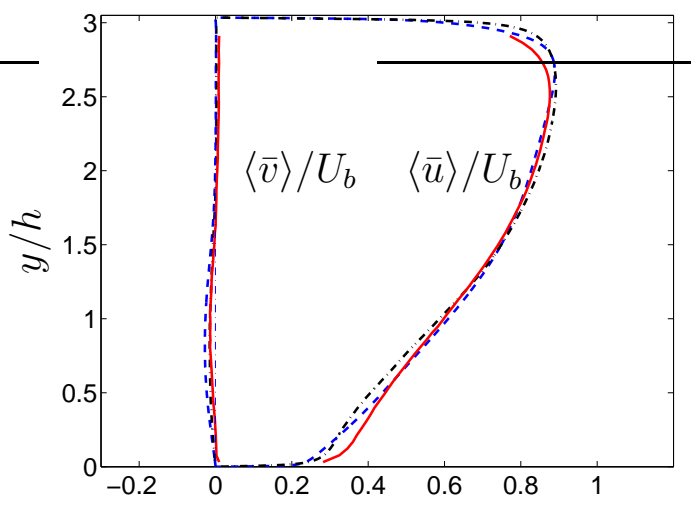

(c) $x / h=6.0$

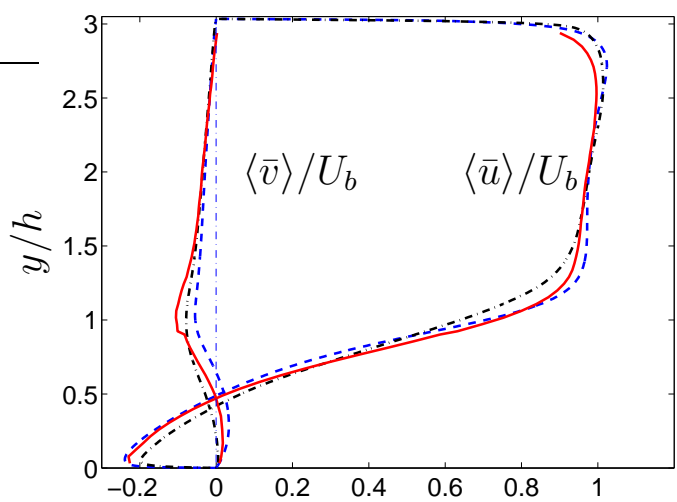

(b) $x / h=2.0$

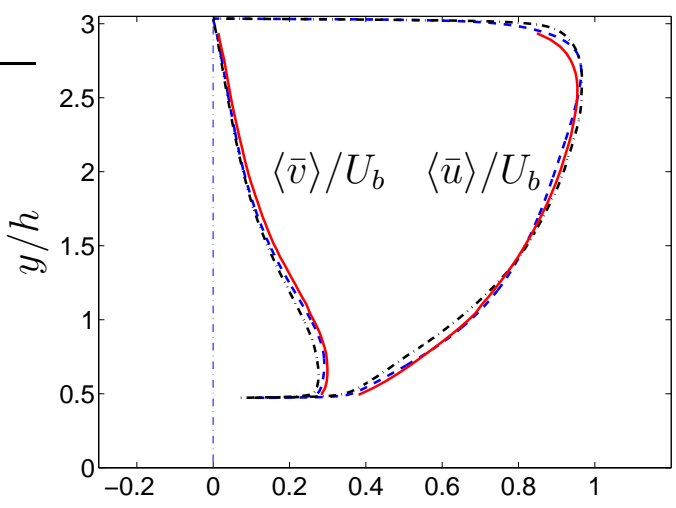

(d) $x / h=8.0$

Figure 23: Periodic hill flow, $R e=37000$ : Comparison of mean velocities. - - - : LRN PANS - $\sigma$ model; - - - : Dynamic Smagorinsky model; — : Experimental data.

the total shear stresses than with the Dynamic Smagorinsky model. This is illustrated in Fig. 25 for the total shear stresses and in Fig. 26 for the modelled $\nu_{u}$. It is interesting to note that the modelled turbulent viscosity is smaller at this high Reynolds number compared to $R e=10600$ (see Fig. 21). With the Dynamic Smagorinsky model, this is attributed to the fact that the 
grid cell size is smaller for $R e=37000$ compared to $R e=10600$. Although the grid cell size does not appear explicitly in the PANS formulation, the LRN PANS $-\sigma$ model has also responded to the finer grid resolution.

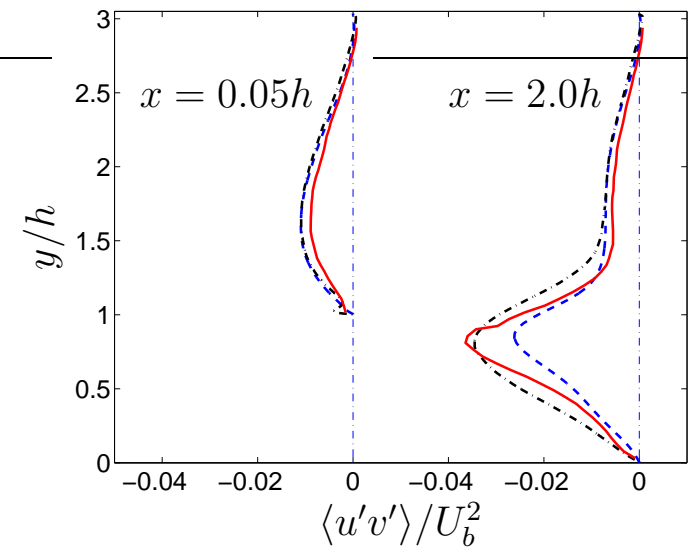

(a) $x / h=0.05$ and 2.0

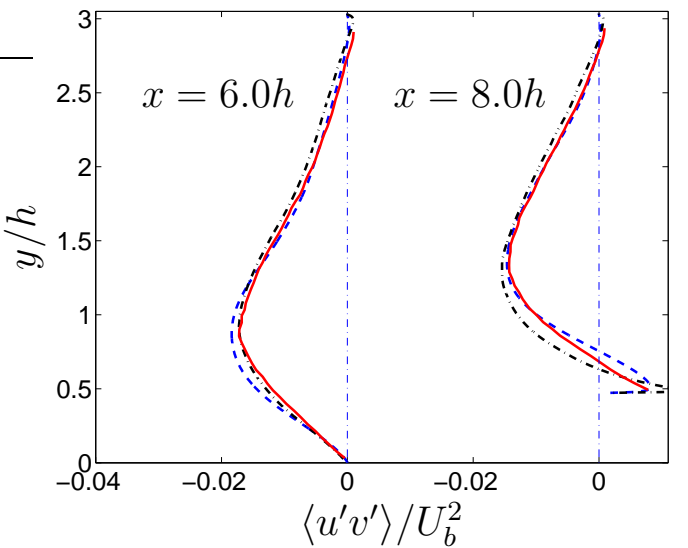

(b) $x / h=6.0$ and 8.0

Figure 24: Periodic hill flow, $R e=37000$ : Profiles of resolved Reynolds shear stresses. - - - : LRN PANS - $\sigma$ model; -. . : Dynamic Smagorinsky model; __ : Experimental data.

\section{Conclusions}

A low-Reynolds-number PANS formulation is proposed in order to improve the near-wall modelling behavior of the original (standard) PANS model. A general LRN form of $k-\varepsilon$ model has been taken as the RANS parent model in the derivation, incorporating viscous and wall-damping effects in the model coefficients. The resulting formulation can thus lead to different LRN PANS variants, upon the choice of the LRN $k-\varepsilon$ base model. In the present work, the AKN LRN model was taken as the platform in the 


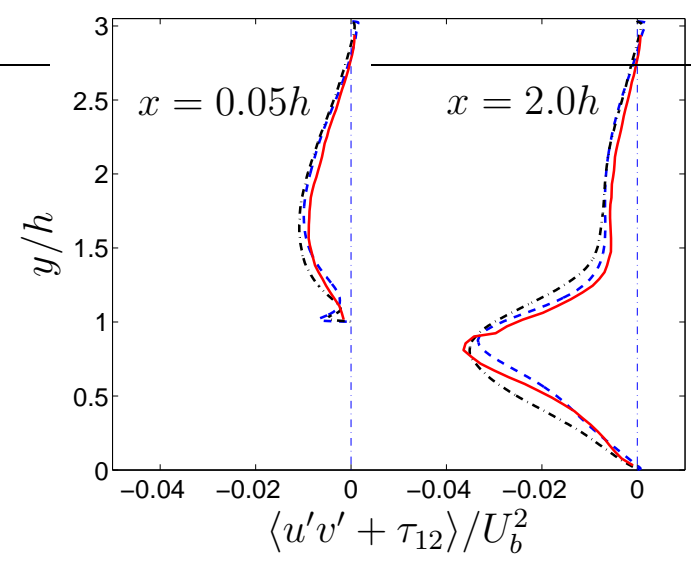

(a) $x / h=0.05$ and 2.0

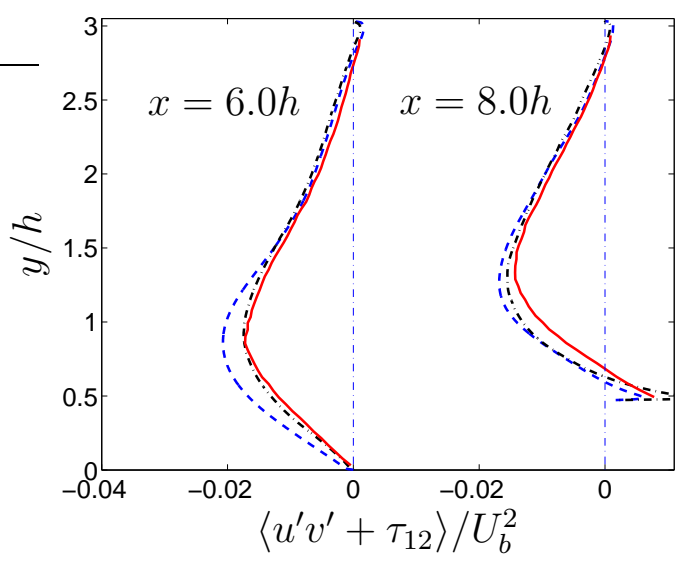

(b) $x / h=6.0$ and 8.0

Figure 25: Periodic hill flow, $R e=37000$ : Profiles of total (i.e. resolved and modelled) turbulent Reynolds shear stresses. - _ _ : LRN PANS - $\sigma$ model; -.. : Dynamic Smagorinsky model; — : Experimental data.

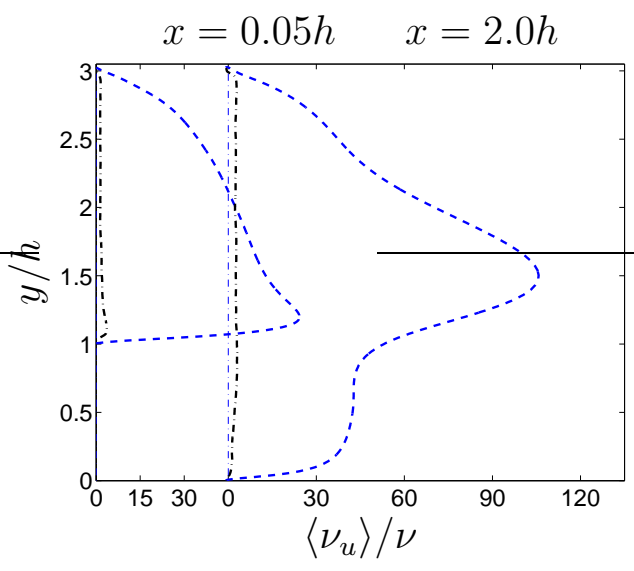

(a) $x / h=0.05$ and 2.0

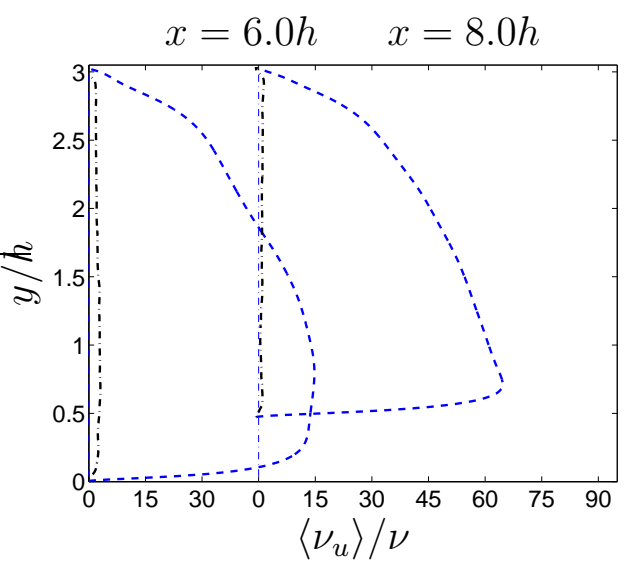

(b) $x / h=6.0$ and 8.0

Figure 26: Periodic hill flow, $R e=37000$ : Modelled turbulent viscosities. LRN PANS- $\sigma$ model. - - - : Dynamic Smagorinsky model.

verification of the LRN PANS modelling performance, in which two damping functions were introduced. Computations were made for, respectively, a 
decaying homogeneous, isotropic turbulence, the turbulent channel flow at $R e_{\tau}=950$, and the periodic hill flow at two different Reynolds numbers at $R e=10600$ and $R e=37000$. For comparison, predictions obtained by the Dynamic Smagorinsky model, have also been included.

One of the main purposes has been to investigate the capabilities of the proposed model in predicting both attached and separated flows, when adopted as a turbulence-resolving modelling approach. With the same grid resolution for the different test cases considered, the effect of the PANS modelling parameter, $f_{k}$, as well as of the PANS turbulent Prandtl number, $\sigma_{k u}$ and $\sigma_{\varepsilon u}$, was also investigated. The results, computed by both the original and the proposed LRN PANS models, have been compared with available DNS, LES or experimental data.

In the computation of DHIT it is shown that the LRN modification imposes insignificant effects, giving a $0.5 \%$ reduction of the destruction term of $\varepsilon_{u}$ for the smallest $f_{k}(=0.2)$ at the fine mesh. Different $f_{k}$ values were evaluated on two grids $\left(32^{3}\right.$ and $\left.64^{3}\right)$. It was found, as expected, that a refined grid requires a reduced value of $f_{k}$ to get the best prediction of energy spectra. When $f_{k}$ is chosen appropriately $\left(f_{k}=0.6\right.$ on the $32^{3}$ grid and $f_{k}=0.2-0.4$ on the $64^{3}$ grid), the LRN PANS is able to produce predictions that are similar to, or better than, the Dynamic Smagorinsky model.

For the channel flow computations on an LES grid, the LRN PANS model 
is able to produce improved predictions for both the mean flow velocity and the resolved turbulence statistics, as compared to the original PANS model. The model also demonstrates a reasonable response to the change of parameter $f_{k}$. In contrast to the inappropriate wall-limiting behavior inherent in the original PANS model, the LRN formulation introduces correct asymptotic properties in the modelled turbulence quantities, which have consequently enabled improved predictions of resolved turbulence statistics in the wall layer. The LRN PANS model is able to give improved predictions, as compared to the Dynamic Smagorinsky SGS model.

The function of the PANS method is well demonstrated for the hill flow. Both the standard and the LRN PANS models produce good predictions for the mean flow and the resolved turbulent quantities. Nonetheless, the improvement resulting from the LRN formulation is sensible and, as intended, this formulation has rendered generally better predictions in near-wall regions than the standard PANS model.

It is noted that the PANS formulation modifies the turbulent diffusion terms in the $k_{u}$ and the $\varepsilon_{u}$ equations, by multiplying a factor of $f_{\varepsilon} f_{k}^{-2}$ to the turbulent Prandtl numbers, namely, $\sigma_{k u}=\sigma_{k} f_{k}^{2} / f_{\varepsilon}$ and $\sigma_{\varepsilon u}=\sigma_{\varepsilon} f_{k}^{2} / f_{\varepsilon}$. Investigation was conducted in the hill flow computation using $\sigma_{k u}=\sigma_{k}$ and $\sigma_{\varepsilon u}=\sigma_{\varepsilon}$ (denoted the LRN PANS $-\sigma$ model) in comparison with the LRN PANS model with $\sigma_{k u}=\sigma_{k} f_{k}^{2} / f_{\varepsilon}, \sigma_{\varepsilon u}=\sigma_{\varepsilon} f_{k}^{2} / f_{\varepsilon}$ and $f_{k}=0.4$. It 
was found that the LRN PANS $-\sigma$ model increases the peaks in $k_{u}$ and $\varepsilon_{u}$ by approximately a factor of five compared to the LRN PANS model and, correspondingly, increases the turbulent viscosity and the modelled Reynolds stresses. The difference in the mean flows predicted by the two models was small. Along with the modelling of sink term in the $\varepsilon$ equation, the option of modifying the turbulent diffusion terms in the modelled turbulence equations is interesting in exploration of turbulence-resolving modelling approaches.

In comparison with LES using the Dynamic Smagorinsky model, the LRN PANS model yields similar or somewhat better results in all three wallbounded flows computed in the present work. Furthermore, it was observed that the required CPU time is slightly less for the LRN PANS model compared to the Dynamic Smagorinsky model. Moreover, the former model is numerically more feasible than the latter which invokes additional testfiltering operations and usually ad-hoc local averaging and/or clipping in the dynamic determination of the model coefficient, particularly when dealing with flows in complex geometries using unstructured grids.

In previous validations and applications, the PANS method has shown encouraging performance for an appreciable range of different flows. Being an engineering modelling approach, this method is based on a unique physical argumentation in deriving its formulation that is different from conventional DES and hybrid models. In searching for improved model-based turbulence- 
resolving approaches, PANS has provided an alternative way, by which a number of available (and relatively robust) RANS models can be readily tested in the framework of the PANS formulation.

As compared to many existing SGS models in LES, the LRN PANS formulation does not invoke the grid cell size, and can thus avoid any ad-hoc determination of the subgrid scale in the model when a stretching/skewing grid is invoked. With this feature, similar to RANS computations, the LRN PANS model may potentially reach a grid-independent solution with successively refined grid at a constant value of $f_{k}$. This feature can help to get rid of unphysical effect of grid arrangement, as being present in some existing turbulence-resolving methods which use the local cell size to justify the RANS-LES switching.

Of the emerging turbulence-resolving modelling approaches, the PANS method is relatively new. Needless to say, much investigation on some of the above and other aspects has to be conducted for further verification and improvement of the modelling. This is however out of the scope of the current work. Using the turbulence-resolving capabilities, the LRN PANS model will in future work be further developed as a zonal method coupled with RANS. The model is presently being used in an embedded LES formulation (Davidson and Peng, 2011). The LRN PANS model offers an elegant way of switching from RANS to LES by setting the value of parameter from 
$f_{k}=1$ to an appropriately small value of $f_{k}$.

\section{Acknowledgments}

The first author was supported by the China Scholarship Council, the Beijing Nature Science Foundation of China (3071002) and the National Nature Science Foundation of China (50779070). The financial support of SNIC (the Swedish National Infrastructure for Computing) for computer time at C3SE (Chalmers Center for Computational Science and Engineering) is also gratefully acknowledged. Part of this project was financed by the EU project ATAAC (Advanced Turbulence Simulation for Aerodynamic Application Challenges), Grant Agreement No. 233710.

\section{References}

Abe, K., Kondoh, T., Nagano, Y., 1994. A new turbulence model for predicting fluid flow and heat transfer in separating and reattaching flows - 1. Flow field calculations. Int. J. Heat Mass Transfer 37, 139-151.

Basara, B., Krajnović, S., Girimaji, S., 2009. PANS vs. LES for computations of the flow around a 3D bluff body. In: 2nd International Conference on Turbulence and Interaction. Martinique, France.

Basu, D., Hamed, A., K.Das, 2007. Assessment of partially averaged navier stokes (PANS) multiscale model in transonic turbulent separated flows. In: 5th Joint ASME/JSME Fluids Engineering Conference. July 30-August 2, San Diego, California USA. 
Chien, K., 1982. Predictions of channel and boundary layer flows with a lowReynolds-number turbulence model. AIAA Journal 20, 33-38.

Comte-Bellot, G., Corrsin, S., 1971. Simple Eularian time correlation of full- and narrow-band velocity signals in grid-generated "isotropic" turbulence. Journal of Fluid Mechanics 48 (2), 273-337.

Davidson, L., 2009. Hybrid LES-RANS: back scatter from a scale-similarity model used as forcing. Phil. Trans. of the Royal Society A 367 (1899), 2905-2915.

Davidson, L., Peng, S.-H., 2003. Hybrid LES-RANS: A one-equation SGS model combined with a $k-\omega$ model for predicting recirculating flows. International Journal for Numerical Methods in Fluids 43, 1003-1018.

Davidson, L., Peng, S.-H., 2011. Emdedded LES with PANS. In: 6th AIAA Theoretical Fluid Mechanics Conference (to be presented). 27-30 June, Honolulu, Hawaii.

Emvin, P., 1997. The full multigrid method applied to turbulent flow in ventilated enclosures using structured and unstructured grids. Ph.D. thesis, Dept. of Thermo and Fluid Dynamics, Chalmers University of Technology, Göteborg.

Frendi, A., Tosh, A., Girimaji, S. S., 2007. Flow past a backward-facing step: Comparison of PANS, DES and URANS results with experiments. International for Computational Methods in Engineering Science and Mechanics 8, 23-38. 
Fröhlich, J., Mellen, C., Rodi, W., Temmerman, L., M.A., Leschziner, 2005. Highly-resolved large eddy simulations of separated flow in a channel with streamwise periodic constrictions. Journal of Fluid Mechanics 526, 19-66.

Germano, M., Piomelli, U., Moin, P., Cabot, W., 1991. A dynamic subgrid-scale eddy viscosity model. Physics of Fluids A 3, 1760-1765.

Girimaji, S., 2005. Partially-Averaged Navier-Stokes model for turbulence: Implementation and Validation. In: 43rd AIAA Aerospace Sciences Meeting and Exhibt. 10-13 January, Reno, Nevada.

Girimaji, S., 2006. Partially-averaged Navier-Stokes model for turbulence: A Reynolds-averaged Navier-Stokes to direct numerical simulation bridging method. ASME Journal of Applied Mechanics 73 (2), 413-421.

Girimaji, S. S., Lavin, T. A., 2006. Investigation of turbulent square jet using PANS method. In: Napolitano, M., Sabetta, F. (Eds.), 44th AIAA Aerospace sciences meeting. Vol. 8. Reno, Nevada, pp. 5860-5870.

Hoffmann, G. H., 1975. Improved from the Low-Reynolds-Number $k-\varepsilon$ turbulence model. Physics of Fluids 618, 309-312.

Hoyas, S., Jimenez, J., 2008. Reynolds number effects on the reynolds-stress budgets in turbulent channels. Physics of Fluids A 20 (101511).

Hsieh, W. D., Chang, K. C., 1996. Calculation of wall heat transfer in pipe- 
expansion turbulent flows. International Journal of Heat and Mass Transfer 39 (18), 3813-3822.

Jones, W., Launder, B., 1972. The prediction of laminarization with a two-equation model of turbulence. Int. J. Heat Mass Transfer 15, 301-314.

Menter, F., Egorov, Y., 2010. The scale adaptive simulation method for unsteady turbulent flow predictions. Part 1: Theory and description. Flow, Turbulence and Combustion 85, 113-138.

Nagano, Y., Tagawa, M., 1990. An improved form of the $k-\varepsilon$ model for boundary layer flows. Journal of Fluids Engineering 112, 33-39.

Rapp, C., 2008. Experimentelle Studie der turbulenten Strömung über periodische Hügeln. Ph.D. thesis, Technical University of Munich, Germany, Germany.

Rapp, C., Manhart, M., 2011. Flow over periodic hills - an experimental study. Experiments in Fluids, DOI:10.1007/s00348-011-1045-y.

Schiestel, R., Dejoan, A., 2005. Towards a new partially integrated transport model for coarse grid and unsteady turbulent flow simulations. Theoretical and Computational Fluid Dynamics 18, 443-468.

Song, C.-S., Park, S.-O., 2009. Numerical simulation of flow past a square cyclinder using Partially-Averaged Navier-Stokes model. Journal of Wind Engineering and Industrial Aerodynamics 97, 37-47. 